\title{
COUPLING OF SLOW AND FAST WAVES \\ TO PLASMAS NEAR THE LOWER \\ HYBRID FREQUENCY
}

BY

R. L. BERGER, F, W. PERKINS, AND $F$, TROYON

\section{PLASMA PHYSICS LABORATORY}

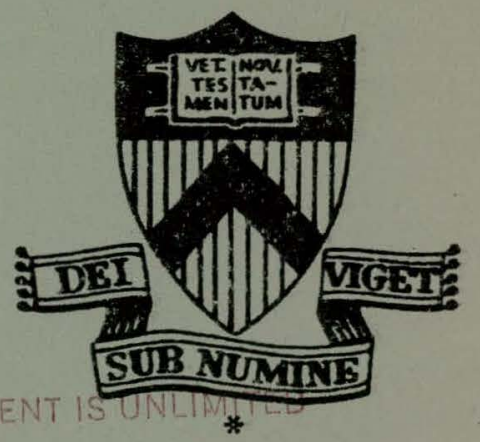

\section{PRINCETON \\ UNIVERSITY PRINCETON, NEW JERSEY}

This work was supported by U. S. Energy Research and Development Administration Contract EY-76-C-02-3073. Reproduction, translation, publication, use and disposal, in whole or in part, by or for the United States Government is permitted. 


\section{DISCLAIMER}

This report was prepared as an account of work sponsored by an agency of the United States Government. Neither the United States Government nor any agency Thereof, nor any of their employees, makes any warranty, express or implied, or assumes any legal liability or responsibility for the accuracy, completeness, or usefulness of any information, apparatus, product, or process disclosed, or represents that its use would not infringe privately owned rights. Reference herein to any specific commercial product, process, or service by trade name, trademark, manufacturer, or otherwise does not necessarily constitute or imply its endorsement, recommendation, or favoring by the United States Government or any agency thereof. The views and opinions of authors expressed herein do not necessarily state or reflect those of the United States Government or any agency thereof. 


\section{DISCLAIMER}

Portions of this document may be illegible in electronic image products. Images are produced from the best available original document. 
NOTICE

This report was prepared as an account of work sponscred by the United States Government. Neither the United States nor the United States Energy Research and Development Administration, nor any of their employees, nor any of their contractors, subcontractors, or their employees, makes any warranty, express

or implied, or assumes any legal liability or

responsibility for the accuracy, completeness or usefulness of any information, apparatus, product or process disclosed, or represents that its use would not infringe privately owned rights.

Printed in the United States of Arnerica.

Available from

National Technical Information Service

U. S. Department of Commerce 5285 Port Royal Road

Springfield, Virginia 22151

Price: Printed Copy $\${ }_{*} ;$ Microfiche $\$ 3.00$

*Pages

$1-50$

$51-150$

$151-325$

$326-500$

501-1000
NTIS

Selling Price

$\$ 4.00$

5.45

7.60

10.60

13.60 


\title{
Coupling of Slow and Fast Waves to Plasmas \\ Near the Lower Hybrid Frequency
}

\author{
R. L. Berger \\ Plasma Physics Laboratory, Princeton University \\ Princeton, New Jèrsey 08540 USA \\ F. W. Perkins \\ National Center for Atmospheric Research \\ Boulder, Colorado 80303. USA \\ and \\ F. Troyon

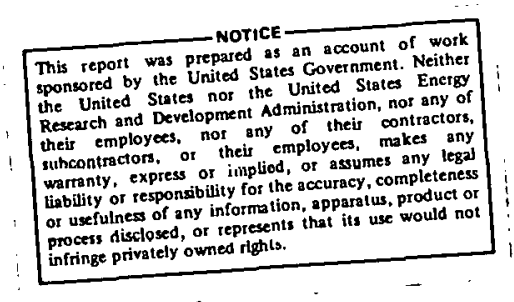 \\ Centre de Recherches en Physique des Plasmas \\ Lausanne, Switzerland
}


Coupling of Slow and Fast Waves to Plasmas

Near the Lower Hybrid Frequency

$$
\text { R. L. Berger }
$$

Plasma Physics Laboratory, Princeton University Princeton, New Jersey 08540 USA

F. W. Perkins

National Center for Atmospheric Research Boulder Colorado 80303 USA

and

$$
\text { F. Troyon }
$$

Centre de Recherches en Physique des Plasmas Lausanne, Switzerland

Lower hybrid frequency heating relies on the efficient coupling of vacuum radiation to plasma waves that propagate across the field lines and heat the plasma core. Penetration through the plasma surface to high densities imposes a minimum value on $\mathrm{N}_{\|}$, the parallel index of refraction. The evanescent layer at low density is very thin for the slow wave, but is much larger for the fast wave, especially for large tokamaks, and thus, short wavelengths will not penetrate. A new effect proportional to the product of the density gradient and the azimuthal wavenumber, can either reduce or increase the evanescence for the fast wave. For hot plasmas, linear electron Landau damping imposes an upper limit on $N_{\|}$so that only a narrow spectrum of waves can penetrate. The coupling efficiency is obtained for plane 


$$
\vdots-2-
$$

waves incident on the plasma and is found to depend weakly on the density gradient scale length. For a range of $\mathrm{N}_{\|}$,' resonant eigenmode solutions are found that are localized to the low density plasma regions.

\section{INTRODUC TION}

The excitation of plasma waves with fxequency noar the lower hybrid frequency is a promising means under consideration to heat tokamak plasmas to thermonuclear temperatures. An attractive feature of this method is that the wave can he launrhed by an array of waveguides, dil all intetallic structure that is important for reactor design. No coils or other structures that are necessary for lowe $\mathrm{r}$ frequency heating schemes are introduced into the vacuum vessel. In contrast to upper hybrid frequency heating, the power is efficiently produced and is currently available at the powers ( $>1$ MW) nèeded to heat reactors. Fxperiments on both small linear devices [1] and tokamaks [2] have shown that heating is most efficient when the frequency is within a few times the maximum lowe $r$ hybrid frequency. The extension of this heating scheme to reactors requires that the waves be launched by antennae, such as waveguides, in the vacuum region outside the central hot plasma. Recently, experiments have shown that minimum reflection of the incident radiation occurs when the band of wavenumbers parallel to the main magnetic field excited by waveguide arrays satisfy accessibility criteria [3]. These results are in qualitative agreement with 
the theoretical calculations of Brambilla [4] for excitation of the slow electrostatic mode. It is well known that there are two modes of oscillation near the lower hybrid frequency in the cold plasma limit - the slow electrostatic mode and the fast electromagnetic whistler mode.

Only the slow electrostatic wave has been used for plasma heating experiments to date, primarily due to technological constraints. To launch the fast wave, an azimuthal electric field is imposed near the plasma boundary; and to satisfy accessibility criteria, the field should be periodic axially with parallel wavelength such that $\mathrm{N}_{\|}>1$. A waveguide cannot satisfy both the se criteria unless it is filled with a dielectric material which is unacceptable for a reactor. Coils could be designed for use in small linear devices; however typical $\omega_{\text {pe }} \ll \omega_{c e}$ and the evanescence layer that the fast wave must penetrate near the boundary is too great.

In this pape $r$ we examine the conditions for slow and fast wave excitation. The fast wave may have advantages in heating over the slow wave in that it does not propagate in the resonance cones characteristic of the slow electrostatic wave [b]; thus, the heating is not highly localized and the electric fields are are not as large for a given input power so that nonlinear processes may be easier to avoid [6].

We present a three-dimensional slab model calculation of the propagation of electromagnetic waves from the vacuum to the plasma interior. We assume that nonlinear effects such as filamentation [7] do not influence wave propagation, i. e., linear wave propagation is assumed. The conditions necessary for propagation and the wave polarization are examined in the low 
density plasma. near the vacuum and in the plasma interior. We solve the WKB aispersion relations in both regions. The important parameters for penetration are $\Omega^{2}=\omega^{2} / \omega_{c e} \omega_{c i}$, the parallel index of refraction $N_{\|}=c \|_{\|} / \omega$, and $\Omega_{p e}^{2}=\omega_{p e}^{2} / \omega_{c e}^{2}$ where $\omega$ is the wave. frequency, $\mathrm{l}_{\|}$is the wavenumber along the magnetic field, $\omega_{\text {pe }}$ is the electron plasma frequency, and $\omega_{c e}\left(\omega_{c i}\right)$ is the electron (ion) gyrofrequency. Penetration to the plasma core is only possible for waves with a parallel index of refraction greater than a minimum value determined by $\Omega^{2}$ and the maximum value of $\Omega_{\text {pe }}^{2}[8]$. On the other hand, waves with $N_{\|}>1$ are evanescent in the vacuum and will spatially attenuate before reaching the propagating region unless the distance from the source to the cutoff density is much less than a vacuum wavelength. In addition, for hot plasmas linear electron Landau damping will

attenuate the wave before it pienetrates to the plasma interior if $\omega / k_{\|} V_{e} \leqslant 3$. This criterion imposes an upper bound on $N_{\|}$that

$$
\mathrm{N}_{\|}<\frac{\left(\mathrm{m}_{\mathrm{e}} \mathrm{c}^{2} / \mathrm{T}_{\mathrm{e}}\right)^{1 / 2}}{3} \simeq 7.5\left(\frac{1 \mathrm{keV}}{\mathrm{T}_{\mathrm{e}}}\right)^{1 / 2}
$$

The best antennae will be designed to effect a compromise between these effects.

Any realistic source will generate a spectrum of wavelengths parallel to the magnetic field $B$ and perpendicular to $B$ and the density gradient. We solve the equations that describe the wave 
propagation from the vacuum to the interior for arbitrary wavenumbers. The WKB solutions in the plasma interior are connected through the cutoff and mode conversion regions by exact solutions of equations valid in each region.

There are no terms that depend explicitly on the density gradient for slow waves near the slow wave cutoff. However, the fast wave cutoff depends on the density gradient, $(1 / n)(d n / d x)$, explicitly if the azimuthal wavenumber $k_{y}$ is finite. Waveguide sources produce a spectrum of azimuthal wavenumbers and this new term, which depends on the sign of $k_{y}(d n / d x)$, moves the cutoff to higher or lower density.

Moreover, we treat the important case [9] when the incoming wave undergoes mode conversion to a wave of another type. As a result, we find the reflection coefficient for each Fourier component of the incident vacuum radiation and find new eigen mode solutions for certain values of $\mathrm{N}_{\mathrm{II}}$.

The organization of the paper is as follows. In Section 2 we present the basic equations, find the WKB dispersion relation, and the accessibility criteria. In section 3 the amplitudes of incident and reflected waves are related to the incident radiation from the vacuum. Finally, in section 4 we discuss the relevance of these results to current and future tokamak heating experiments near the lower hybrid frequency. 


\section{BASIC EQUATIONS AND ACCESSIBILITY}

The analysis is carried out within the framework of the two fluid equations and a slab plasma. The plasma is assumed homogeneous in the $\mathrm{y}$ and $\mathrm{z}$ direction but has a density increasing in the $x$ direction. A uniform magnetic field is assumed in the $z$ direction. The waves under consideration have frequency between

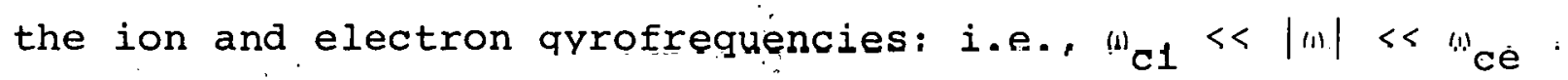
Because the density varies over a large range, the frequency is comparable with the electron plasma frequency at the surface and the ion plasma frequency in the interior. The ions are treated as unmagnetized; the electrons are considered strongly magnetized. Thermal corrections and damping effects are neglected because of the long wavelength nature of the radiation. The propagation of these waves are described by the equations,

$$
\begin{gathered}
\left(-N_{\|}^{2}-N_{y}^{2}+\varepsilon_{\perp}(x)\right) E_{x}-i\left(N_{y} \frac{\partial}{\partial \xi}+\varepsilon_{x y}\right) E_{y}-i N_{\|} \frac{d}{d \xi} E_{z}=0 \\
\left(-N_{\|}^{2}+\varepsilon_{\perp}(x)+\frac{\partial^{2}}{\partial \xi^{2}}\right) E_{y}-i\left(N_{y} \frac{\partial}{\partial \xi}-\varepsilon_{x y}\right) E_{x}+N_{\|} N_{y} E_{z}=0 \\
\left(\frac{d^{2}}{d \xi^{2}}-N_{y}^{2}+\varepsilon_{\|}\right) E_{z}-i N_{\|} \frac{d}{d \xi} E_{x}+N_{\|} N_{y} E_{y}=0
\end{gathered}
$$


that are valid throughout the density profile. We define

$$
\begin{aligned}
\varepsilon_{\perp}(x) & =1+\frac{\omega_{p e}^{2}}{\omega_{c e}^{2}}-\frac{\omega_{p i}^{2}}{\omega^{2}} \\
& "=\frac{\omega_{p e}^{2}}{\omega \omega_{c e}} \\
\varepsilon_{X \bar{Y}} & = \\
\varepsilon_{\|} & =1-\frac{\omega_{p e}^{2}}{\omega^{2}}
\end{aligned}
$$

and $\mathrm{N}_{\mathrm{y}}=\mathrm{ck}_{\mathrm{y}} / \omega, \mathrm{N}_{\|}=\mathrm{ck}_{\|} / \omega$, and $\xi=\omega \mathrm{x} / \mathrm{c}$. The two modes of propagation described by Eqs. (1) - (3) are the fast wave or whistler wave which is fully electromagnetic and the slow wave which is primarily electrostatic. The fast wave has most of its energy in the magnetic components and, if $\omega^{2}<\omega_{p e}^{2}$, it is polarized such that the electric field vector points in the $\mathbf{x}$ direction and the magnetic field vector lies in the $y-z$ plane. The slow wave has roughly half its energy in the field and half in particles; it is polarized such that its electric field vector lies in the $x-z$ plane. The fast (slow) wave can be launched by imposing a field at the plasma boundary with the electric field vector in the $\mathrm{y}$ direction (z direction).

The WKB dispersion relation, obtained by assuming an elkonal solution of Eqs. (1) $-(3), E(x)=\bar{E} \exp \left[i \int^{x} k(x) d x\right\rfloor$, is 


$$
\begin{aligned}
& \varepsilon_{\perp} N^{4}+N^{2}\left[\left(N_{H}^{2}-\varepsilon_{\perp}\right)\left(\varepsilon_{\perp}+\varepsilon_{\|}-2 N_{y}^{2}\right)+\varepsilon_{x y}^{2}+2 N_{H}^{2} N_{Y}^{2}\right] \\
& +\left(\varepsilon_{\|}:-N_{y}^{2}\right)\left[\left(N_{y}^{2}+N_{H}^{2}-\varepsilon_{\perp}\right)\left(N_{\|}^{2}-\varepsilon_{\perp}\right)-\varepsilon_{x y}^{2}\right] \\
& +\mathrm{N}_{\|}^{2} \mathrm{~N}_{\dot{y}}^{2}\left(\mathrm{~N}_{\mathrm{Y}}^{2}+\mathrm{N}_{\|}^{2}-\varepsilon_{\perp}\right)=0
\end{aligned}
$$

The solutions to Eq. (4) can be examined, in some useful limits. In the vacuum, $\varepsilon_{x y}=0, \varepsilon_{\perp}=\dot{\varepsilon}_{\|}=1 ;$ and $\mathrm{N}^{2}=1-\mathrm{N}_{\|}^{2}-\mathrm{N}_{\mathrm{Y}}^{2}$. Waves with $\mathrm{N}^{2}>0$ propagate and with $\mathrm{N}^{2}<0$ do not. There are two cutoff densities where $\mathrm{N}^{2}=0$. The density at cutoff is

$$
\omega_{p e}^{2}\left(x_{s}\right)=\omega^{2}\left(N_{y}^{2}+N_{\|}^{2}-1\right) /\left(N_{\|}^{2}-1\right)
$$

for the slow: wave and

$$
\omega_{p e}^{2}\left(x_{f}\right):=\omega \omega_{c e}\left(N_{y}^{2}+N_{\|}^{2}-i\right)^{1 / 2}\left(N_{\|}^{2}-1\right)^{1 / 2}
$$

for the fast wave. At higher densities beyond the fast and slow 
wave cutoffs $\varepsilon_{x y}^{2} \simeq\left|\varepsilon_{||}\right| \simeq m_{i} / m_{e}$, whereas $\varepsilon_{\perp} \simeq N_{\|}^{2} \sim N_{y}^{2} \sim 1$ and the roots of $\mathrm{Eq}$. (4) are

$\mathrm{N}_{\mathrm{s}, \mathrm{f}}^{2}=\frac{\omega_{\mathrm{pe}}^{2}}{2 \omega^{2} \varepsilon_{\perp}}\left\{\mathrm{N}_{11}^{2}-\varepsilon_{\perp}-\frac{\omega_{\mathrm{pe}}^{2}}{\omega_{\mathrm{ce}}^{2}} \pm\left[\left(\mathrm{N}_{\|}^{2}-\varepsilon_{\perp}-\frac{\omega_{\mathrm{pe}}^{2}}{\omega_{\mathrm{ce}}^{2}}\right)^{2}-4 \varepsilon_{\perp} \frac{\omega_{\mathrm{pe}}^{2}}{\omega_{\mathrm{ce}}^{2}}\right]^{1 / 2}\right\}$

provided $\mathrm{N}_{11}^{2}-1 \gg \Omega\left(\mathrm{m}_{\mathrm{e}}^{\prime} / \mathrm{m}_{i}\right)^{1 / 2}$. The two roots are equal when the quantity under the radical in Eq. (7) vanishes. The two densities for which such equality holds are given by $\omega_{\mathrm{pi}}^{2}\left(\mathrm{x}_{\mathrm{c}}\right)=\omega_{ \pm}^{2}$ where

$\omega_{ \pm}^{2}=\omega^{2}\left[1-\left(1-2 \Omega^{2}\right) N_{\|}^{2} \pm 2 N_{\|} \Omega\left(1-N_{\|}^{2}+\Omega^{2} N_{\|}^{2}\right)^{1 / 2}\right]$.

clearly, such a density exists if $\Omega^{2}>1$ or if $\Omega^{2}<1$ and $\mathrm{N}_{\| 1}^{2}<\left(1-\Omega^{2}\right)^{-1}$.

We first consider $\Omega^{2}>1$. In this case, $\varepsilon_{\perp}>0$ for all densities and $\mathrm{N}^{2}>0$ (propagation) if $\mathrm{N}_{\|}^{2}>\sqrt{\varepsilon_{\perp}}+\omega_{\mathrm{pe}} / \omega_{\mathrm{ce}} \cdot$ It is easy to show this is only possible if $\omega_{\mathrm{pi}}^{2}<\omega_{-}^{2}$. Therefore, for $\Omega^{2}>1, \omega_{-}^{2}$ is the ion plasma frequency at the maximum density to which either a fast or slow wave can penetrate. This behavior is shown qualitatively in Fig. la. 
If $1 / 2<\Omega^{2}<1$ and $N_{\|}^{2}<\Omega^{2}\left(1-\Omega^{2}\right)^{-1}$, then $N^{2}<0$ for both roots at $\omega_{\mathrm{pi}}^{2}(\mathrm{x})=\omega_{+}^{2}$. At $\omega_{\mathrm{pi}}^{2}=\omega^{2} /\left(1-\Omega^{2}\right)=\omega_{\mathrm{r}}^{2}, \mathrm{~N}_{\mathrm{s}}^{2}$ changes sign but $\mathrm{N}_{\mathrm{f}}^{2}$ does not. Thus, the slow wave propagates on the high density side of the resonance! This case is shown in Fig. $1 \mathrm{~b}$. Between $\omega_{-}^{2}$ and $\omega_{+}^{2}$, the wave is a strongly decaying oscillation. If $1 / 2<\Omega^{2}<1$ and $\Omega^{2}\left(1-\Omega^{2}\right)^{-1}<N_{\|}^{2}<\left(1-\Omega^{2}\right)^{-1}, N^{2}>0$ at $\omega_{\mathrm{pi}}^{2}=\omega_{+}^{2} \cdot$ This case is sketched in Fig. 1c. Between $\omega_{-}^{2}$ and $\omega_{+}^{2}$ the wave is a decaying oscillation. The wave propagates between $\omega_{+}^{2}$ and $\omega_{r}^{2}$ where the slow wave undergoes mode conversion to a hot plasma wave. At higher densities, the slow wave is again evanescent; the fast wave continues to propagate. It is clear from Eq. (7) that the imaginary part of $k_{x}$ between $\omega_{-}^{2}$ and $\omega_{+}^{2}$ is large so that unless the two densities almost coincide, i.e., $\mathrm{N}_{\| 1}^{2} \simeq\left(1-\Omega^{2}\right)^{-1}$, the damping is too strong for significant tunneling to occur.

No turning points exists if $\mathrm{N}_{\|}^{2}>\left(1-\Omega^{2}\right)^{-1}$ and $\Omega^{2}<1$. This case is shown in Fig. 1d. The slow wave has a resonance at $\omega_{\mathrm{pi}}^{2}=\omega_{\mathrm{r}}^{2}$ where it converts to a hot plasma wave. When $\Omega^{2}>1$ and $\mathrm{N}_{\|}^{2}<\left(1-\Omega^{2}\right)^{-1}$, an incident fast (slow) wave mode converts to an outgoing slow (fast) wave at $x=x_{-}$. In the next section we will show how to connect these two waves. If $\Omega^{2}>\Omega_{\mathrm{lh}}^{2}=\Omega_{\mathrm{pe}}^{2} /\left(1+\Omega_{\mathrm{pe}}^{2}\right)$, the condition that the wave penetrate to this density is either (1) that $\mathrm{N}_{\|}^{2}>\left(1-\Omega^{2}\right)^{-1}$ because, if $\Omega^{2}<1$, no turning points exist, i.e., both branches are distinct for all densities, or (2) that the sign of the term under the radical in Eq. (7) be positive. If $\Omega^{2}>1$, the 
second criterion applies. If $\Omega^{2}<1$, the least restrictive criterion applies. The two criteria are identical for the frequency,

$$
\Omega_{0}^{2}=-\frac{\Omega_{\mathrm{pe}}^{2}}{2}+\left(\Omega_{\mathrm{pe}}^{2}+\frac{\Omega_{\mathrm{pe}}^{4}}{4}\right)^{1 / 2}
$$

and the condition, $\mathrm{N}_{\|}^{2}>\left(1-\Omega^{2}\right)^{-1}$, is less restrictive if $\Omega<\Omega_{0}$. The accessibility criteria is then $\mathrm{N}_{\|}>\mathrm{N}_{\|}^{*}$ where

$$
\begin{gathered}
\mathrm{N}_{\|}^{*}=\mathrm{N}_{\|}^{+}=\left(1-\Omega^{2}\right)^{-1 / 2} \text { if } \Omega_{l \mathrm{~h}}^{2}<\Omega^{2}<\Omega_{0}^{2} \\
\mathrm{~N}_{\|}^{*}=\left(\Omega_{\text {pe }}+\sqrt{\varepsilon_{\perp}}\right) \text { if } \Omega^{2}>\Omega_{0}^{2} .
\end{gathered}
$$

We now know the conditions that are necessary for wave propagation in the plasma interior. The results are conveniently characterized by the normalized frequency $\Omega$ and the parallel index of refraction $\mathrm{N}_{\text {II }}$ - In all cases, the wave is evanescent in the plasma if $\mathrm{N}_{\|}^{2}<1$. For $\mathrm{N}_{\|}>1$, we discuss the case $\Omega<1$ and $\Omega>1$ separately. If $\Omega>1$, then there is a maximum 
density to' which a slow or fast wave propagates at which point the wave converts to the other polarization. If $\Omega<1$, another parameter enters the problem; i.e., $\mathrm{N}_{\|}^{+}$. If $\mathrm{N}_{\|}>\mathrm{N}_{\|}^{+}$, the two branches of the dispersion relation remain distinct except in the vacuum and the slow wave has a resonance $\left(k_{x} \rightarrow \infty\right)$ at $\omega_{\ell h}(x)=\omega \cdot$ For $1<N_{\|}<N_{||}^{+}$, the slow or fast wave propagates from low densities to a density where mode conversion to the wave of the other polarization occurs. At still higher densities, the wave again propagates and the slow wave has a resonance at $\omega_{\ell h}(x)=\omega !$ However, the distance between densities for which the wave propagates is typically too large for significant tunneling to occur.

\section{WÄVE EQUATION SOLUTIONS AND CONNECTION FORMULAE}

In the previous section, we derived the WKB dispersion relation for wave propagation in the vicinity of the lower hybrid resonance and identified the cütoffs, mode conversions, and resonances $\left(\mathrm{N}^{2} \rightarrow \infty\right)$. In this section, we find the connection formulae that enable one to relate the amplitude of a plane wave incident on the plasma from the vacuum to the amplitude of the wave in the plasma interior. If the wave is cutoff to the right of the turning point, the high density side of the cutoff, the correct boundary condition is that the amplitude vanish at $\xi \rightarrow \infty$. For waves that propagate to the right of the cutoff, outgoing wave boundary conditions are appropriate unless $\mathrm{N}_{\|}<\mathrm{N}_{\|}^{*}$ in which 
case both outgoing and incoming waves are allowed due to mode conversion at higher density. In this latter case, a standing wave in the radial direction can be set up for certain values of $\mathbf{N}_{11}$

As a result of this analysis, we can find the reflection and transmission coefficients for any incident plane wave. It is then a straightforward calculation to find the reflection and transmission coefficients for a wave produced by any launching structure because it can be expressed as a superposition of plane waves. We specify the electric fields parallel to the magnetic field, $E_{\|}$, and perpendicular to $\underline{B}_{O}$ and the density gradient, $E_{y}$, in the vacuum region,

$$
\left(\begin{array}{c}
E_{y}\left(x, k_{y}, k_{I I}\right) \\
E_{\| 1}\left(x, k_{y}, k_{I I}\right)
\end{array}\right)=\left(\begin{array}{c}
\sigma_{y} \\
\sigma_{z}
\end{array}\right) \exp \left[-N_{0}\left(\xi-\xi_{w}\right)\right]+\left(\begin{array}{c}
\rho_{y} \\
\rho_{z}
\end{array}\right) \exp \left[N_{0}\left(\xi-\xi_{w}\right)\right]
$$

where $\mathrm{N}_{\mathrm{O}}^{2}=\mathrm{N}_{\mathrm{Y}}^{2}+\mathrm{N}_{\|}^{2}-1$ and $\mathrm{N}_{\mathrm{O}} \rightarrow-\mathrm{in} \mathrm{N}_{\mathrm{O}}$ if $\mathrm{N}_{\mathrm{O}}^{2}<0$. Here $\xi_{\mathrm{w}}$ is the position of the source. The coefficients $\sigma_{y}$ and $\sigma_{z}$ can be found as Fourier integrals of the electric fields $E_{y}(x, y, z)$ and $E_{z}(x, y, z)$ specified at $\xi=\xi_{w}$.

In Fig. 2, we show the model of the density profile. The magnetic field variation is small in the regions of interest. 
The plasma density begins a steep rise to its central value at $\xi=\xi_{a}$. To the left of the plasma surface $\left(\xi=\xi_{a}\right)$, the density is taken to be zero. This assumption has no practical effect on the results for fast wave launching but this model does not treat the possible existence of a tenuous plasma in this region which would be sufficient to support propagation of the slow wave. The slow wave and fast wave cutoffs are located at $\xi=\xi_{\mathrm{p}}$ and $\xi_{\mathrm{f}}=\xi_{\mathrm{F}}$ respectively. For those waves that do not meet the accessibility criteria, a mode conversion layer is located at $\xi=\xi_{\mathrm{C}}$.

In the plasma interior, we can describe the slow and fast waves by the WKB solutions,

$$
\begin{aligned}
E_{X}= & {\left[N_{S}(\xi)\right]^{1 / 2}\left\{E_{S 1} \exp \left[i P_{S}(\xi)\right]+E_{S 2} \exp \left[-i P_{S}(\xi)\right]\right\} } \\
& \\
& +\left[N_{f}(\xi)\right]^{1 / 2}\left\{E_{f 1} \exp \left[i P_{f}(\xi)\right]+E_{f 2} \exp \left[-i P_{f}(\xi)\right]\right\}
\end{aligned}
$$

where

$$
\begin{aligned}
P_{\mathbf{S}}(\xi)= & \int_{\xi_{\mathrm{p}}}^{\xi} \mathrm{N}_{\mathbf{S}}\left(\xi^{\prime}\right) \mathrm{d} \xi^{\prime} \\
& \vdots \\
\mathrm{P}_{\mathrm{f}}(\xi)= & =\int_{\xi_{f}}^{\xi} \cdot \mathrm{N}_{f}\left(\xi^{\prime}\right) \mathrm{d} \xi^{\prime}
\end{aligned}
$$


and $\mathrm{N}^{2}=\mathrm{c}^{2} \mathrm{k}_{\mathrm{x}}^{2} / \omega^{2}$, and $\xi_{\mathrm{p}}$ and $\xi_{\mathrm{f}}$ are the positions of the slow and fast wave cutoffs respectively. In this region, the slow wave is a backward wave in the $x$ direction so that $E_{s 2}$ represents a wave propagating away from the plasma boundary; the fast wave is a forward wave so $\mathrm{E}_{\mathrm{fl}}$ represents a wave propagating away from the boundary.

3a. Solutions Near the slow wave Cutoff

In the very low density region near the slow wave cutoff, we make the approximations that $\varepsilon_{\perp}=1, \varepsilon_{x y}=0$, and as a result obtain the equations

$$
\begin{aligned}
& N_{0}^{2} E_{x}+i N_{Y} \frac{d}{d \xi} E_{y}+i N_{\|} \frac{d}{d \xi} E_{z}=0, \\
& \left(\frac{d^{2}}{d \xi^{2}}-N_{0}^{2}\right)\left[\left(1-N_{\|}^{2}\right) E_{Y}+N_{\|} N_{y} E_{\|}\right]=0, \\
& \left(\frac{a^{2}}{d \xi^{2}}+Q_{S}^{2}(\xi)\right) E_{\|}=0,
\end{aligned}
$$

where $\mathrm{N}_{0}^{2}=\mathrm{N}_{\| 1}^{2}+\mathrm{N}_{\mathrm{y}}^{2}-1$ and

$$
\mathrm{Q}_{\mathrm{s}}^{2}(\xi)=\left(\frac{\omega_{\mathrm{pe}}^{2}}{\omega^{2}}-1\right)\left(\mathrm{N}_{\|}^{2}-1\right)-\mathrm{N}_{\mathrm{y}}^{2}
$$


From Eqs. (16) and (17), we see that the presence of finite $\mathrm{N}_{\mathrm{Y}}$ moves slow wave cutoff to higher density; i.e., the cutoff now occurs at $\xi=\xi_{p}$ where

$$
\omega_{p e}^{2}=\omega^{2}\left(N_{y}^{2}+N_{\| 1}^{2}-1\right) /\left(N_{\| 1}^{2}-1\right) .
$$

The waves with $\mathrm{N}_{\|}^{2}>1$ propagate in the plasma region and are evanescent in the vacuum region; those with $\mathrm{N}_{11}^{2}+\mathrm{N}_{\mathrm{Y}}^{2}<1$ are evanescent: in plasma and propagate in the vacuum region; those with $\mathrm{N}_{\|}^{2}+\dot{\mathrm{N}}_{\mathrm{Y}}^{2}>1$ but $\mathrm{N}_{\|}^{2}<1$ are evanescent everywhere. The solution to Eq. (14) is

$$
E_{y}=\frac{N_{\|} N_{y}}{N_{\|}^{2}-1} E_{\|}+e_{1} \exp \left(+N_{0} \xi\right)+e_{2} \exp \left(-N_{0} \xi\right)
$$

The first term is just the small $y$ component of the slow wave; the other terms are the fast wave components which behave essentially as if they are still in the vacuum. For the waves that are cutoff at $\xi=\xi_{p}$ we expand $Q_{S}^{2}(\xi)$ in a Taylor series about this point; i.e.,

$$
Q_{s}^{2}(\xi)=\alpha_{s}\left(\xi-\xi_{p}\right),
$$

where 


$$
\alpha_{s}=\frac{\left(N_{\|}^{2}-1\right)}{\omega^{2}} \frac{c}{\omega}\left(\frac{d}{d x} \omega_{p e}^{2}(x)\right)_{x=x_{p}},
$$

and find the solution to the resultant Airey equation to be

$$
E_{z}=a_{s}{ }^{A i}\left[-\lambda_{s}\left(\xi-\xi_{p}\right)\right]+b_{s} B i\left[-\lambda_{s}\left(\xi-\xi_{p}\right)\right],
$$

if $\mathrm{N}_{\mathrm{II}}^{2}+\mathrm{N}_{\mathrm{y}}^{2}>1$ and

$$
E_{z}=a_{s}^{\prime} A i\left[+\lambda_{s}\left(\xi-\xi_{p}\right)\right]+b_{s}^{\prime} B i\left[\lambda_{s}\left(\xi-\xi_{p}\right)\right],
$$

if $\mathrm{N}_{\|}^{2}+\mathrm{N}_{\mathrm{y}}^{2}<1$ where $\lambda_{\mathrm{s}}^{3}=\left|\alpha_{\mathrm{s}}\right|$. Here $\mathrm{Ai}(\mathrm{x})$ and $\mathrm{Bi}(\mathrm{x})$ are Airey's functions [10]. In the second case, the condition that $E_{z}$ be finite as $\xi \rightarrow \infty$ requires $b_{s}^{\prime}=0$. If $N_{\|}^{2}+N_{y}^{2}>1$ and $\mathrm{N}_{11}>\mathrm{N}_{\|}^{*}$, then Eq. (19) should connect smoothly to the WKB solutions (11) with $\mathrm{E}_{\mathrm{sl}}=0$; i.e., an outgoing slow wave. Thus, we obtain the condition $b_{s}=i a_{s}$ and $2 a_{s}=-r_{s}^{*} E_{s 2}$. If $N_{\| 1}^{2}>1$ and $\mathrm{N}_{\|}<\mathrm{N}_{\|}^{*}$, we must include the possibility of outgoing waves due to mode conversion of incoming fast waves at $\xi=\xi_{c} \cdot$ Using Eq. (14) to find the slow wave components of $\mathrm{E}_{\mathrm{X}}$ and matching this solution asymptotically to the WKB solution, we establish the relations, 


$$
\begin{aligned}
& a_{s}+i b_{s}=r_{s} E_{s l}, \\
& a_{s}-i b_{s}=-r_{s}^{\star} E_{s 2},
\end{aligned}
$$

where

$$
I_{S}=\frac{2 \sqrt{\pi}\left(\mathrm{N}_{\|}^{2}-1\right) \exp (+\pi i / 4)}{\mathrm{N}_{\|}\left|\alpha_{\mathrm{S}}\right|^{1 / 6}},
$$

and $r_{s}^{*}$ denotes the complex conjugate of $r_{s:}$. The remaining components with $\mathrm{N}_{\|}^{2}<1$ and $\mathrm{N}_{\|}^{2}+\mathrm{N}_{\mathrm{y}}^{2}>1$ which are evanescent everywhere will be assumed to obey the vacuum solution everywhere.

3b. Solutions Near the Fast Wave Cutoff

The fast wave has a cutoff at higher density than the slow wave. In the vicinity of the fast wave cutoff, the slow wave components can be treated in the WKB sense, and the remaining fast wave components obey the equations:

$$
\begin{gathered}
N_{0}^{2} E_{x}+i\left(N_{y} \frac{d}{d \xi}+\varepsilon_{x y}\right) E_{y}=0 \\
E_{H} \simeq 0 \\
\left(\frac{d^{2}}{d \xi^{2}}+Q_{F}^{2}(\xi)\right) E_{y}=0
\end{gathered}
$$


where

$$
Q_{F}^{2}=\left[\varepsilon_{x y}^{2}-\varepsilon_{x y}^{\prime} N_{Y}-N_{O}^{2}\left(N_{\| 1}^{2}-1\right)\right] /\left(N_{\|}^{2}-1\right)
$$

and $\varepsilon_{\mathrm{xy}}^{\prime}=\mathrm{d} \varepsilon_{\mathrm{xy}} / \mathrm{d} \xi$. The fast wave cutoff occurs at a density such that

$$
\omega_{p e}^{4}=\omega^{2} \omega_{c e}^{2} N_{o}^{2}\left(N_{\|}^{2}-1\right)
$$

if $\mathrm{N}_{\mathrm{Y}}=0$. However, if $\mathrm{N}_{Y} \neq 0$, the cutoff occurs at higher densities if $\mathrm{k}_{\mathrm{y}}(\mathrm{dn} / \mathrm{dx})>0$ and lower densities if $\mathrm{k}_{\mathrm{y}}(\mathrm{dn} / \mathrm{dx})<0$. Using the definition $\varepsilon_{x y}=\omega_{p e}^{2} / \omega \omega_{c e}$, we see that the density gradient dependent term in Eq. (26) is important if

$$
\delta=\frac{N_{y} \varepsilon^{\prime} y}{\varepsilon_{x y}^{2}}=\frac{\omega \omega_{c e}}{\omega_{p e}^{2}} N_{y} \frac{c}{\omega L_{f}} \sim 1,
$$

where $L_{f}^{-I}=d / d x$ lnn and this term is evaluated at the density defined by Eq. (6). For the purpose of assessing the importance of this term, we assume a parabolic density dependence on the radius $r$ of a tokamak; i.e., $n(r)=n_{0}\left(1-r^{2} / a^{2}\right)$, where $a$ is the minor radius. Then using Eq. (69), we find

$$
\delta=\left|\frac{N_{y} \varepsilon_{x y}^{I}}{\varepsilon_{x y}^{2}}\right| \sim\left(\frac{2 c}{\omega a}\right) \frac{\omega}{\omega_{c i}} \frac{w^{2} N_{y}}{\left(N_{I I}^{2}-1\right) N_{0}^{2}}
$$


where $\mathrm{w}^{2}=\omega_{\mathrm{pi}}^{2}(\mathrm{r}=0) / \omega^{2} \sim 1$ and $\mathrm{x}=\mathrm{a}-\mathrm{r}$. Because $\mathrm{w} \sim \mathrm{I}$ by assumption and $\omega_{\text {pe }} \sim \omega_{\text {ce }}$ at $r \simeq 0$ for a typical tokamak, we find $\omega / \omega_{c i} \simeq\left(m_{i} / m_{e}\right)^{1 / 2}$. For $n \leq 10^{14}$, this implies $\dot{\omega} \simeq 10^{10}$ and thus we, arrive at the estimate

$$
\delta \simeq\left(\frac{25 \mathrm{~cm}}{\mathrm{a}}\right) \frac{10 \mathrm{w}^{2} \mathrm{~N}_{\mathrm{y}}}{\left(\mathrm{N}_{11}^{2}-1\right) \mathrm{N}_{\mathrm{O}}^{2}} \sim 0.4 \mathrm{w}^{2}
$$

for $\mathrm{a} \sim 50 \mathrm{~cm}, \mathrm{~N}_{\mathrm{y}} \sim 1$, and $\mathrm{N}_{\|} \sim 2$. The slower components of the wave (larger $N_{11}$ ) are less affected by this effect, but these waves have a larger evanescent region through which to propagate and thus a well-designed antenna will not put much energy in these Fourier components. This term is important only in the vicinity of the fast wave cutoff: at higher densities $\varepsilon_{\mathrm{xy}}^{2} \gg\left|\varepsilon_{\mathrm{xy}}^{1}\right| \mathrm{N}_{\mathrm{y}}$ and at lower densities $\left|\varepsilon_{\mathrm{xy}}^{1}\right| \mathrm{N}_{\mathrm{y}} \ll \mathrm{N}_{0}^{2}\left(\mathrm{~N}_{\|}^{2}-1\right)$. Thus, the basic description of wave propagation afforded by the WKB dispersion relation remains valid in the presence of this effect.

We will, ignore this effect in the remainder of this paper (i.e., we set $\varepsilon_{x y}^{l}=0$ ) because even though $\delta \sim 1$ at the turning point, $\delta \ll 1$ at slightly higher densities and lower densities and thus the distance from the plasma boundary to the turning point will not change significantly. It is this distance compared to the free space wavelength, that will have the most influence 
on the ability of a wave to penetrate. It should be emphasized, though, that, in smaller tokamaks for which a $25 \mathrm{~cm}, \delta>1$ because the density gradients at low density will be larger. It is this type of tokamak in which fast wave heating should be tried first and a quantitative theory of the coupling of an antenna to plasma should include these terms.

We verify that the slow wave can be treated in the WKB sense in this region by using Eq. (69) for the density gradient and the WKB wavenumber in the $x$ direction,

$$
\mathrm{k}_{\xi}=\frac{c \mathrm{k}_{\mathrm{x}}}{\omega}=\frac{{ }^{\omega} \mathrm{pe}}{\omega}\left(\mathrm{N}_{\|}^{2}-1\right)^{1 / 2} \simeq\left(\frac{{ }^{\omega} \mathrm{ce}}{\omega}\right)^{1 / 2}\left(\mathrm{~N}_{\|}^{2}-1\right)^{3 / 4} \mathrm{~N}_{\mathrm{O}}^{1 / 2}
$$

we obtain

$$
k_{x} L_{f}=17 \Omega^{-1 / 2}\left(\frac{m_{p}}{m_{i}}\right)^{1 / 2} \frac{\left(N_{11}^{2}-1\right)^{5 / 4} N_{0}^{3 / 2}}{w^{2}}\left(\frac{a}{50 \mathrm{~cm}}\right)\left(\frac{B}{50 \mathrm{kG}}\right) \text {. }
$$

Under most conditions, $k_{x} L_{f} \gg 1$.

The division into propagating and evanescent modes is the same as for slow waves with respect to $\mathrm{N}_{\mathrm{Il}}$ and $\mathrm{N}_{\mathrm{Y}}$ - Taylor expanding about the point $\xi_{F}$ where $Q_{F}^{2}=0$ we obtain the Airey equation with solutions 


$$
E_{y}=a_{F} A_{i}\left[-\lambda_{F}\left(\xi-\xi_{F}\right)\right]+b_{F} B_{i}\left[-\lambda_{F}\left(\xi-\xi_{F}\right)\right]
$$

if $\mathrm{N}_{\|}^{2}>1$ and

$$
E_{Y}=a_{F}^{1} A_{i}\left[\lambda_{F}\left(\xi-\xi_{F}\right)\right]+b_{F}^{1} B_{i}\left[\lambda_{F}\left(\xi-\xi_{F}\right)\right]
$$

if $\mathrm{N}_{\|}^{2}+\mathrm{N}_{\mathrm{Y}}^{2}<1$. Here $\lambda_{F}=\left|\alpha_{F}\right|^{1 / 3}$ and

$$
\alpha_{F}=\left(\frac{d Q_{F}(\xi)}{d \xi}\right)_{\xi=\xi_{F}}=\frac{2 \omega_{p e^{2}}^{\left(\xi_{F}\right)}}{\omega^{2} \omega_{C e}^{2}}\left(\frac{d}{d \xi} \omega_{p e}^{2}\right)_{\xi=\xi_{F}}
$$

The second term in Eq. (29) is exponentially increasing for $\xi>\xi_{F}$. The condition that $E_{Y}$ be finite as $\xi . \rightarrow$ requires $\mathrm{b}_{\mathrm{F}}^{l}=0$. The boundary conditions on Eq. (28) depend on $\mathrm{N}_{i l} / \mathrm{NN}_{\|}^{*}$. If $\mathrm{N}_{\|}>\mathrm{N}_{\|}^{*}$, Eq. (28) should describe an outgoing wave as $\xi \rightarrow+\infty$ and thus $b_{F}=-i a_{F}$ since this wave is a forward wave in the $\xi$ direction. If $I<\mathrm{N}_{\|}<\mathrm{N}_{\|}^{*}$, both outgoing and incoming fast wave components exist because a mode conversion occurs at $\omega_{\mathrm{pi}}=\omega_{-}$. The determination of the relation between $b_{\mathrm{F}}$ and $a_{F}$ awaits the solution of the wave equation in the mode conversion region. In summary, we have the relations:

$$
\mathrm{b}_{\mathrm{F}}^{1}=0, \quad \mathrm{~N}_{\mathrm{II}}^{2}+\mathrm{N}_{\mathrm{Y}}^{2}<1
$$




$$
\begin{aligned}
b_{F} & =-i a_{F}, N_{\|}^{2}+N_{Y}^{2}>1, N_{\| l}>N_{\|}^{*}, \\
2 b_{F} & =r_{f} E_{f l}
\end{aligned}
$$

and that

$$
\begin{aligned}
& b_{F^{\prime}}-i a_{F^{\prime}}=r_{F^{\prime}} F_{F^{\prime} l}, \\
& b_{F}+i a_{F}=-r_{F}^{*} E_{F 2},
\end{aligned}
$$

for $\mathrm{N}_{\mathrm{II}}^{2}+\mathrm{N}_{\mathrm{Y}}^{2}>1$ and $\mathrm{N}_{\|}<\mathrm{N}_{\|}^{*}$ where

$$
r_{F}=\frac{2 i \sqrt{\pi} N_{O}^{2} \exp (-\pi i / 4)}{\lambda_{F}^{1 / 2}\left[\left(N_{I I}^{2}-1\right)^{1 / 2}+i N_{Y}\right]}
$$

3c. Solutions in Mode Conversion Region

If $N_{Y}^{2}+N_{\|}^{2}>1$ and $N_{\|}<N_{\|}^{*}$, the slow and fast waves convert into one another and the WKB solutions are no longer valid in the vicinity of the mode conversion point $\xi=\xi_{c}$. The equation that adequately describes both slow and fast waves is

$$
\left(\frac{d^{4}}{d \xi^{4}}+b \frac{d^{2}}{d \xi^{2}}+c\right) E_{x}=0
$$


The corresponding WKB wavenumber dispersion equation has the solution

$$
\mathrm{N}^{2}=\frac{\mathrm{b}}{2} \pm \frac{1}{2}\left(\mathrm{~b}^{2}-4 \mathrm{c}\right)^{1 / 2}
$$

where the upper (lower) sign corresponds to the slow (fast) waves asymptotically. The coefficients b and $c$ in Eq. (36) can be obtained by identifying the corresponding terms in Eq. (7). The mode conversion point corresponds to the vanishing of the second term in Eq. (36). Thus, we expand about $\xi=\xi_{c}$ and obtain

$$
b^{2}-4 c=-4 \alpha_{c}\left(\xi-\xi_{c}\right)
$$

and Eq. (28) becomes

$$
\left[\left(\frac{d^{2}}{d \xi^{2}}+\frac{b}{2}\right)^{2}+\alpha_{c}\left(\xi-\xi_{c}\right)\right] E_{x}=0
$$

Because $b \gg \alpha_{c}\left|\xi-\xi_{c}\right|$, we remove the fast $\xi$ variation by the substitution

$$
E_{x}=\exp \left(i \frac{b}{\sqrt{2}}\left(\xi-\xi_{c}\right)\right) \psi_{+}+\exp \left(-i \frac{b}{\sqrt{2}}\left(\xi-\xi_{c}\right)\right) \psi_{-},
$$


and obtain the approximate second order equation

$$
\left(\frac{d^{2}}{d r^{2}}-r\right) \psi_{ \pm}=0
$$

where $r=\lambda_{c}\left(\xi-\xi_{c}\right)$ and $\lambda_{c}^{3}=2 b / \alpha$. Equation (39) has the usual Airey function solutions and thus

$$
\psi_{ \pm}=a_{ \pm}^{\prime} A i\left[\lambda_{c}\left(\xi-\xi_{c}\right)\right]+b_{ \pm} B i\left[\lambda_{c}\left(\xi-\xi_{c}\right)\right] .
$$

Since we have assumed that, in the case $\Omega^{2}<1$, the distance between $\omega_{-}^{2}$ and $\omega_{+}^{2}$ is too large for the wave to tunnel through, the wave should be a decaying oscillation to the right of $\xi=\xi_{c}$. This requires $b_{ \pm}=0$. Matching the solution in Eq. (38) to the WKB solutions in Eq. (11), we establish the connection formuale,

$$
\begin{aligned}
& E_{s 1}=E_{f 1} \exp i \Phi \\
& E_{F 2}=E_{s 2} \exp i \Phi
\end{aligned}
$$

where

$$
\Phi=\int_{\xi_{F}}^{\xi_{C}} N_{F}(\xi) d \xi-\int_{\xi_{p}}^{\xi} N_{S}(\xi) d \xi-\frac{\pi}{2}
$$


Now the reflected wave amplitudes for both slow and fast waves can be found in the plasma boundary region in terms of the incident amplitudes. We summarize the results by the matrix equation,

$$
\left(\begin{array}{c}
b_{c} \\
u_{F}
\end{array}\right)=\left(\begin{array}{cc}
h_{11} & h_{12} \\
\cdot & \\
h_{21} & h_{22}
\end{array}\right)\left(\begin{array}{c}
a_{j} \\
\cdot \\
a_{F}
\end{array}\right)
$$

where $\underset{\simeq}{h}=0$ if $N_{y}^{2}+N_{\| 1}^{2}<1, h_{11}=i, h_{22}=-i, h_{12}=h_{21}=0$ if $\mathrm{N}_{\|}^{2} \simeq \mathrm{N}_{\mathrm{Y}}^{2}>1$ and $\mathrm{N}_{\|}>\mathrm{N}_{\|}^{*}$. If $\mathrm{N}_{\|}^{2}+\mathrm{N}_{\mathrm{Y}}^{2}>1$ and $\mathrm{N}_{\|}<\mathrm{N}_{\|}^{*}$,

$$
\begin{aligned}
& h_{11}=-h_{22}=-\tan \left(\Phi+\alpha_{N}\right) \\
& h_{12}=-\left|\frac{r_{s}}{r_{f}}\right| \sec \left(\Phi+\alpha_{N}\right) \\
& h_{21}=\left|\frac{r_{f}}{r_{S}}\right| \sec \left(\Phi+\alpha_{N}\right)
\end{aligned}
$$

where $\sin \alpha_{N}=N_{Y} / N_{O}$. Note that for propagating waves 
$\left(\mathrm{N}_{11}^{2}>1\right)$ the determinant of the matrix $\stackrel{\mathrm{h}}{=}$ is unity. For convenience, we assume that between the plasma and the wave launcher is a vacuum region. In this region, electromagnetic waves that couple to the plasma waves previously are given by Eq. (11). We impose continuity of the magnetic field components $\mathrm{B}_{y}$ and $\mathrm{B}_{z}$ at $\xi=\xi_{\mathrm{a}}$, which is justified provided the density goes smoothly to zero or, less restrictively, if $\omega_{\text {pe }}^{2}\left(\xi_{a}\right) \ll \omega^{2}$. These four equations allow us to find $\rho_{y}$ and $\rho_{z}$ in terms of $\sigma_{y}$ and $\sigma_{z}$. These solutions can be expressed by the equation

$$
\left(\begin{array}{l}
\rho_{z} \\
\rho_{Y}
\end{array}\right)=\left(\begin{array}{ll}
y_{11} & y_{12} \\
& \\
y_{21} & y_{22}
\end{array}\right)\left(\begin{array}{l}
\sigma_{z} \\
\sigma_{y}
\end{array}\right) \quad \exp \left[-2 N_{0}\left(\xi_{a}-\xi_{W}\right)\right]
$$

where, if $\mathrm{N}_{\mathrm{II}}^{2}+\mathrm{N}_{\mathrm{y}}^{2}>1$,

$$
\begin{aligned}
y_{11}= & -G\left[\left(g_{1}^{\prime}-N_{o} g_{1} / \lambda_{s}\right)\right] \\
& \left.\left(g_{2}^{\prime}+N_{o} g_{2} / \lambda_{F}\right)-h_{12} h_{21} g_{3}^{-} g_{4}^{+}\right], \\
y_{22}= & -G\left[\left(g_{2}^{\prime}-N_{o} g_{2} / \lambda_{F}\right)\right. \\
& \left.\left(g_{1}^{\prime}+N_{o} g_{1} / \lambda_{s}\right)-h_{12} h_{21} g_{3}^{+} g_{4}^{-}\right],
\end{aligned}
$$




$$
\begin{aligned}
& \mathrm{y}_{21}=-2 \mathrm{~h}_{21} \mathrm{G}\left(\mathrm{N}_{\mathrm{O}} / \pi \lambda_{\mathrm{S}}\right), \\
& \mathrm{y}_{12}=-2 \mathrm{~h}_{12} \mathrm{G}\left(\mathrm{N}_{\mathrm{O}} / \pi \lambda_{\mathrm{F}}\right),
\end{aligned}
$$

and

$$
\mathrm{G}^{-1} \equiv\left(\mathrm{g}_{1}^{\prime}+\mathrm{N}_{\mathrm{O}} \mathrm{g}_{1} / \lambda_{\mathrm{s}}\right)\left(\mathrm{g}_{2}^{\prime}+\mathrm{N}_{\mathrm{o}} \mathrm{g}_{2} / \lambda_{\mathrm{F}}\right)-\mathrm{h}_{12} \mathrm{~h}_{21} \mathrm{~g}_{3}^{+} \mathrm{g}_{4}^{-}
$$

We have made the definitions

$$
\begin{gathered}
g_{1}=\left[A i(z)+h_{11} B i(z)\right]_{z=\lambda_{S}}\left(\xi_{p}-\xi_{a}\right) \\
g_{2}=\left[A i(z)+h_{22} B i(z)\right]_{z=\lambda_{F}}\left(\xi_{F}-\xi_{a}\right) \\
\vdots \\
g_{3}^{ \pm}=\left[[d B i(z)] / d z \pm N_{0} B i(z) / \lambda_{s}\right\}_{z=\lambda_{S}}\left(\xi_{p}-\xi_{a}\right) \\
\vdots \\
g_{4}^{ \pm}=\left[d /(d z) B i(z) \pm N_{O} B i(z) / \lambda_{F}\right]_{z=\lambda_{F}}\left(\xi_{F}-\xi_{a}\right)
\end{gathered}
$$

and $g_{1}^{\prime}=\mathrm{dg}_{1} / \mathrm{dz}$ and $\mathrm{g}_{2}^{\prime}=\mathrm{dg}_{2} / \mathrm{dz}$. If $\mathrm{N}_{11}^{2}+\mathrm{N}_{\mathrm{y}}^{2}<1, \mathrm{y}_{12}=\mathrm{y}_{21}=0$ and

$$
\begin{gathered}
y_{11}=\left(\frac{\left|N_{o}\right|+i(d / d \xi) \ln A i\left[\lambda_{s}\left(\xi-\xi_{s}\right)\right]}{\left|N_{0}\right|-i(d / d \xi) \ln A i\left[\lambda_{s}\left(\xi-\xi_{s}\right)\right]}\right)_{\xi=\xi_{a}}, \\
y_{22}=\left(\frac{\left|N_{O}\right|+i(d / d \xi) \ln A i\left[\lambda_{f}\left(\xi-\xi_{F}\right)\right]}{\left|N_{O}\right|-i(d / d \xi) \ln A_{i} i\left[\lambda_{f}\left(\xi-\xi_{F}\right)\right]}\right)_{\xi=\xi_{a}}
\end{gathered}
$$


If $\mathrm{N}_{\|}^{2}+\mathrm{N}_{\mathrm{Y}}^{2}>1$ and $\mathrm{N}_{\|}^{2}<1$, no propagation is possible in vacuum or plasma and we set $y_{11}=y_{22}=y_{12}=y_{21}=0$. We have now completed the description of the wave propagation in plasma and the coupling of these waves to the vacuum.

For the purpose of computing the efficiency of coupling of a source to the plasma, we assume a sheet current located at $\xi=\xi_{w}$ having components $j_{y}$ and $j_{z}$. We assume the vacuum wall is located several parallel wavelengths away from the source and impose the boundary condition for wave with $\mathrm{N}_{0}^{2}>0$ that $\mathrm{E}_{\mathrm{Y}}=\mathrm{E}_{\mathrm{H}}=0$ at $\xi=-\infty$. The work done on the plasma is proportional to the imaginary part of $\underline{E} \cdot \dot{j}$ or to the resistive impedance. The inductive impedance of the source without the plasma is proportional to

$$
\underline{\sigma} \cdot \dot{j}^{*}=\frac{4 \pi i \omega}{2 \omega^{2} N_{o}}\left[\left(N_{\| l}^{2}-1\right)\left|j_{\|}\right|^{2}+\left(N_{y}^{2}-1\right)\left|j_{y}\right|^{2}+2 N_{\|} N_{y} R_{e}\left(j_{y} j_{\|}^{*}\right)\right]
$$

and the impedance due to the plasma is 


$$
\begin{aligned}
& -30- \\
& \underline{\rho} \cdot \dot{j}^{*}=-\frac{4 \pi i \omega}{2 \omega^{2} N_{0}} \exp \left[-2 N_{o}\left(\xi_{a}-\xi_{w}\right)\right]\left\{\left[N_{\|}^{2}-1\right) y_{\|}+N_{\|} N_{y} y_{12}\right]|j \||^{2} \\
& +\left[\left(N_{y}^{2}-1\right) y_{22}+N_{1 i} N_{y} y_{2 l}^{2}\right]\left|j_{y}\right|^{2} \\
& +\left[y_{\|} N_{\|} N_{y}+\left(N_{Y}^{2}-1\right) y_{12}\right] j_{\|}^{*} j_{Y} \\
& \left.+\left[\left(N_{\| 1}^{2}-1\right) y_{21}+N_{\|} N_{y} y_{22}\right] j_{\|} j_{y}^{*}\right\}
\end{aligned}
$$

In any given: application of Eq. (57), we would choose $j_{Y}=0$ for excitation of the slow wave and $j_{\|}=0$ for excitation of the fast wave. For waves that satisfy the accessibility criteria, $y_{12}=y_{21}=0$, and one can consider the impedance for fast or slow wave excitation separately. Dividing the plasma loaded impedance term (57) by the unloaded impedance term (56), we have the impedance for slow wave excitation;

$$
\mathrm{z}_{\mathrm{s}}=-\exp \left[-2 \mathrm{~N}_{\mathrm{O}}\left(\xi_{\mathrm{a}}-\xi_{\mathrm{w}}\right)\right] \mathrm{y}_{\| 1}
$$

and the impedance for fast wave excitation

$$
\mathrm{z}_{\mathrm{f}}=-\exp \left[-2 \mathrm{~N}_{\mathrm{o}}\left(\xi_{\mathrm{a}}-\xi_{\mathrm{w}}\right)\right] \mathrm{y}_{22}
$$


Provided $\mathrm{N}_{\|}>\mathrm{N}_{\|}^{*}$ and $\mathrm{N}_{\|}^{2}>1$, the impedance is bounded. For $1<N_{\|}<N_{\|}^{*}$, eigenmode exist and this fact is reflected in the impedance by poles in G defined in Eq. (52). The coupling is best when the resistive impedance $R_{s, f}=I m z_{s, f}$ is a maximum. One expects zero resistive impedance if the distance from the plasma surface, $\xi=\xi_{a}$, to the turning point $\xi_{\mathrm{p}}$ or $\xi_{\mathrm{f}}$ is too large; i.e., if $z_{s}=\lambda_{s}\left(\xi_{p}^{\prime}-\xi_{a}\right)>1$ or $z_{f}=\lambda_{f}\left(\xi_{f}-\xi_{a}\right)>>1$. In this limit $\mathrm{Bi}(\mathrm{z})>\mathrm{Ai}(\mathrm{z})$ and $\mathrm{R}_{\mathrm{S}, \mathrm{f}} \simeq \mathrm{Ai} / \mathrm{Bi} \sim 0$.

The penalty in placing the source too far from the plasma is clear from the exponential factor in (57) and (58). We assume that $N_{0}\left(\xi_{a}-\xi_{w}\right) \ll 1$ and examine the dependence of $Z_{s, f}$ on $N_{\text {II }}$ in the limit $\lambda_{s}\left(\xi_{p}-\xi_{a}\right) \ll 1$ and $\lambda_{f}\left(\xi_{f}-\xi_{a}\right) \ll 1$, in which case

$$
z_{s, f}=\frac{\beta \exp (2 i \psi)+N_{o} / \lambda_{s, f}}{-\beta \exp (2 i \psi)+N_{o} / \lambda_{s, f}}
$$

where $B=0.72901$ and $\psi=\pi / 3$. The resistive impedance is

$$
z_{j}=\frac{\sqrt{3} x_{j}}{x_{j}^{2}+1+x_{j}} \text {, }
$$

where $x_{\mathbf{s}}=\mathrm{N}_{\mathrm{o}} / \lambda_{\mathbf{s}} \beta$ and $\chi_{\mathbf{f}}=\mathrm{N}_{\mathrm{o}} / \lambda_{f^{\beta}}$. The maximum value of 
$\mathrm{z}_{j}=3^{-1 / 2}=0.58$ occurs when $x_{j}=1$. The optimum matching occurs for a particular combination of wavelengths and density gradients. In the next section; we will examine these points in more detail. The impedance for $1<\mathrm{N}_{\|}<\mathrm{N}_{\|}^{*}$ has poles which correspond to the resonant excitation of eigenmodes. The eigenmodes exist when the path between the slow and fast wave cutoff through the mode conversion point is an integral number of wavelengths. It is easy to show in either the small or large argument limit of the Airey functions that the eigenvalue condition is equivalent to $\tan \phi=\tan \Phi$ or

$\Phi=-\frac{\pi}{2}+\int_{\xi_{f}}^{\dot{\xi}_{\mathrm{f}}^{\mathrm{c}} \mathrm{c}} \mathrm{N}_{\mathrm{f}}(\xi) \mathrm{d} \xi-\int_{\xi_{\mathrm{p}} \mathrm{c}}^{\xi_{\mathrm{p}}} \mathrm{d} \xi_{\mathrm{S}}(\xi)=\left(\mathrm{n}+\frac{1}{2}\right) \pi+\Phi_{0}$

where $\Phi_{0}=0^{i}$ in the large argument limit and $\Phi \sim \pi / 4$ in the small argument limit. The impedance for a real plasma is bounded either due to the natural damping of the modes or due to convection of wave energy out of the finite region in which the exciting structure is localized.

\section{TOKAMAK APPLICATIONS}

It is clear from the previous sections that only those waves that satisfy the accessibility criteria expressed in Eqs. (9) and (10) and penetrate to the high density region of a tokamak do 
useful heating. Table I gives the value of $N_{\| l}^{*}$ for $\Omega_{\mathrm{pe}}^{2}=\omega_{\mathrm{pe}}^{2} / \omega_{\mathrm{ce}}^{2}$ between 0.2 and 2.0 and $\omega=m_{1 h}, m=1,1.5,2, \infty$. From Table $\dot{I}$, we see that $N_{\|}^{*}$ is an increasing function of both $\Omega_{\text {pe }}^{2}$ and $\omega / \omega_{1 h}$ and, as they increase, accessibility becomes increasingly difficult. Waveguides offer the most attractive means to launch waves into the plasma and they excite a wide band of $N_{\|}$. The width of the spectrum can be narrowed by adjusting the waveguides such that adjacent waveguides have a $180^{\circ}$ phase difference. For example, a slow wave can be launched by constructing an array of waveguides in the $\mathrm{TE}_{1,0}$ mode such that the electric field of the waveguide field is aligned with the magnetic field in the plasma. If the end of the waveguide is 
flush with the wall, the field at the boundary is given by

$$
E_{z}\left(x_{w}, y, z\right)=\sum_{n=1}^{N} \exp \left(i \theta_{n}\right) E_{n} \sin \left(\frac{\pi}{L_{y}} y\right)
$$

for $0<\mathrm{z}<\mathrm{z}_{\mathrm{i}}: 0<\mathrm{y}<\mathrm{I}_{\mathrm{y}} \because$ where $\mathrm{z}_{\mathrm{n}}=\mathrm{nL}_{\mathrm{z}}$ and $E_{z}\left(x_{w}, y, z\right)=0$, otherwise. Here $\theta_{n}$ is the phase of the nth wavequide, in the array and $E_{n}$ is the ampliture nf the field in the nth array. A sketch of such an array is shown in Fig. 3 . Propagation of the $\mathrm{TE}_{10}$ mode in the waveguide reguires that $\omega L_{y} / c<\pi$. Fourier decomposing this field would give in the absence of plasma the coefficient of the incident plane wave

$$
\begin{aligned}
\sigma_{\|}= & \frac{4 \pi L_{y} L_{z}}{\pi^{2}-k_{y}^{2} I^{2} y} \exp \left(\frac{-i k_{y} L_{y}}{2}-\frac{i k_{\|} L_{z}}{2}\right) \cos \left(\frac{k_{y} L_{y}}{2}\right) \frac{\sin \left(k_{\|} L_{z} / 2\right)}{k_{\|} L_{z}} \\
& \frac{1-(-1)^{N} \exp \left(-i N k_{\|} L_{z}\right)}{1+\exp _{1}\left(-i k_{\|} L_{z}\right)}
\end{aligned}
$$

For $N=1,\left|\sigma_{\|}\right|$is peaked at $k_{\|}=k_{y}=0$ with halfwidths $\Delta_{k_{\|}} \sim \pi / 2 L_{z}$ and $\Delta_{k_{Y}} \sim \pi / 2 L_{y} \cdot$ Since $L_{y}$ is fixed by the frequency $L_{y}<\pi c / \omega$, we see that the inçident wave will have a spread in 
$\mathrm{N}_{\mathrm{y}} \sim 1$, which justifies our choice of $\mathrm{N}_{\mathrm{y}} \sim 1$ in estimating the importance of the density gradient terms near the fast wave cutoff. If $N>1$ and even $\sigma_{\|}=0$ at $k_{\|}=0$ and peaks at $k_{\| 0}=\pi / L_{z}$ with halfwidth $\dot{\Delta}_{\mathbf{k}_{\|}}=\pi / \mathrm{NL}_{\mathbf{z}}$. The peak remains at $\mathrm{k}_{\|}=\pi / \mathrm{L}_{\mathrm{z}}$ as $\mathrm{N}$ increases but the width is narrowed. To ensure that most of the spectrum will satisfy the accessibility criteria requires the lower hand of $\mathrm{N}_{\|}$be accessible

$$
\mathrm{N}_{110}-\Delta \mathrm{N}_{10}=\frac{c k}{\omega}-\frac{c \Delta \mathrm{k}_{\|}}{\omega} \geq \mathrm{N}_{\|}^{\star}
$$

or equivalently

$$
\frac{c \pi}{\omega L_{z}}>\frac{N}{N-1} N_{H}^{*}
$$

There are two upper bounds on $\mathrm{N}_{11}:$ (1) The shorter wavelength waves decay spatially at a faster rate in the evanescent region between the antenna and the cutoff, (2) the shorter wavelengths are absorbed by the plasma before they propagate to the interior. The former effect is reflected in the model discussed in Sec. 3 by the attentuation factor $A_{t}$ in the incident wave [see Eq. (11)]

$$
A_{t}=\exp \left[-2 N_{0}\left(\xi_{a}-\xi_{w}\right)\right]
$$


and by the arguments of the Airey functions $z_{s}$ and $z_{f}$ which enter the computation of the impedance $\mathrm{Z}$ in Eq. (57). The attenuation factor can be compensated for by moving the antenna forward until $N_{0}\left(\xi_{a}-\xi_{w}\right)<: 1$. The arguments of the Airey functions that enter into the computation of the impedance depend on the distance between the plasma boundary and the cutoff density, the density gradient, and the parallel wavenumber as indicated in Eq. (53). As, we noted at the end of Sec. 3, if $z_{s}=\lambda_{s}\left(\xi_{p}-\xi_{a}\right)$. $>1$, the resistive impedance for slow waves $R_{S} \simeq 0$ and if $z_{f}=\lambda_{f}\left(\xi_{f}-\xi_{a}\right)>>1, R_{f}=0$. A specific model of the density dependence on radius in a tokamak will allow us to compute the upper, bound on $\mathrm{N}_{\| 1}$ in order that $z_{s} \leqslant 1$ and $z_{f} \leq 1$. For $n(r)=n_{0}\left(1,-r^{2} / a^{2}\right)$ where $a$ is the plasma radius, we obtain the approximate expression valid at low densities,

$$
\omega_{\mathrm{pe}}^{2}=\frac{2}{\mathrm{a}}(\mathrm{x}-\mathrm{a}) \frac{\mathrm{m}_{\mathrm{i}}}{\mathrm{m}_{\mathrm{e}}} \omega^{2} \mathrm{w}^{2}
$$

where $\mathrm{w}^{2}=\omega_{\mathrm{p} i}^{2}(\mathrm{r}=0) / \omega^{2}<1$ and $\mathrm{x}=\mathrm{a}-\mathrm{r}$. The density gradient at the slow cutoff is

$$
\frac{\omega L_{p}}{c}=\eta_{p} \frac{\mathrm{N}_{\mathrm{o}}^{2}}{\left(\mathrm{~N}_{\mathrm{H}}^{2}-1\right)}
$$

where 


$$
n_{p}=\frac{m_{e}}{m_{i}}\left(\frac{w a}{2 c}\right) w^{-2}=0.03\left(\frac{a}{50 c m}\right)\left(\frac{m_{e}}{m_{i}}\right)\left(\frac{f}{1 G H z}\right) w^{-2},
$$

and $\mathrm{L}_{\mathrm{p}}^{-1}=\{\mathrm{d} \log [\mathrm{n}(\mathrm{x})] / \mathrm{d} \mathrm{x}\}_{\mathrm{x}=\mathrm{x}_{\mathrm{p}}}$. The density gradient at the fast wave cutoff is

$$
\frac{w L_{f}}{c}=2 \eta_{f}\left(N_{I I}^{2}-1\right)^{1 / 2} N_{o}
$$

where

$$
\begin{aligned}
n_{f} & =\left(\frac{\omega_{c i^{a}}}{4 c}\right) w^{-2} \\
& =0.20\left(\frac{m_{p}}{m_{i}}\right)\left(\frac{a}{50 c m}\right)\left(\frac{B}{50 k G}\right) w^{-2}
\end{aligned}
$$

and $L_{f}^{-1}=[d \log (x) / d x]_{x=x_{f}}$. The effective perpendicular indices of refraction $\lambda_{f}$ for the fast wave and $\lambda_{s}$ for the slow wave have the values:

$$
\begin{aligned}
& \lambda_{s}=N_{0}^{2 / 3}\left(c / \omega L_{p}\right)^{1 / 3}=n_{p}^{-1 / 3}\left(N_{11}^{2}-1\right)^{1 / 3}, \\
& \lambda_{f}=N_{0}^{2 / 3}\left(N_{\|}^{2}-i\right)^{1 / 3}\left(2 c / \omega L_{f}\right)^{1 / 3}=\eta_{f}^{-1 / 3} N_{o}^{1 / 3}\left(N_{\|}^{2}-1\right)^{1 / 6} .
\end{aligned}
$$


Because we have: approximated the parabolic density profile by a linear function near $r \sim a$, the distances $\xi_{p}-\xi_{a}=\omega L_{p} / c$ and $\xi_{f}-\xi_{a}=\omega L_{f} / c^{:}$, and, thus, we arrive at the expression for the arguments of the Airey functions

$$
z_{s}=\lambda_{s}\left(\xi_{p_{i}}-\xi_{a}\right)=\eta_{p}^{2 / 3} N_{o}^{2} /\left(N_{11}^{2}-1\right)^{2 / 3}
$$

and

$$
z_{f}=\lambda_{f}\left(\xi_{f}-\xi_{a}\right)=2 n_{f}^{2 / 3}\left(N_{\|}^{2}-1\right)^{2 / 3} N_{O}^{4 / 3} .
$$

Evidently, evanescense imposes no restriction on $N_{\|}$for the slow wave. However, for the fast wave, the condition that $z_{f}<1$ imposes the restriction if $\mathrm{N}_{\mathrm{y}}<\mathrm{N}_{\|}$, that

$$
\mathrm{N}_{11}^{2}<1,+\left(0.32 \frac{\mathrm{m}_{\mathrm{p}}^{2}}{\mathrm{~m}_{i}^{2}}\left(\frac{\mathrm{a}}{50 \mathrm{~cm}}\right)^{2}:\left(\frac{\mathrm{B}}{50 \mathrm{kG}}\right)^{2} \frac{1}{\mathrm{w}^{4}}\right)^{-1 / 4}
$$

If $\mathrm{a}=50 \mathrm{~cm}, \mathrm{~B}=50 \mathrm{kG}, \mathrm{W}=\mathrm{I}$ and a hydrogen plasma, or $\mathrm{W}=1 / \sqrt{2}$ and a deuterium plasma:, this restricts $\mathrm{N}_{\|}<1.53$. This upper limit is not as sensitive a function of the magnetic field as $N_{\|}^{*}$, the critical $N_{\|}$required for accessibility. Thus, even though the upper bound on $\mathrm{N}_{11}$ given by Eq. (72) decreases with magnetic field, the optimum condition for fast wave accessibility is $\Omega_{\text {pe }}<1$; i.e., strong magnetic fields. 
The second limit imposed on $\mathrm{N}_{\|}$is due to absorption of the wave by linear electron Landau damping or nonlinear processes. In the former case, the condition is that $\omega / k_{\|} v_{e}>3$ where $v_{e}=\left(T_{e} / m_{e}\right)^{1 / 2}$. We choose three as the limit because, for higher phase velocities, even relatively modest amounts of energy can flatten the parallel electron velocity distribution to render Landau damping ineffective. The condition $\omega / k_{\|} v_{e}>3$ can be translated to the condition

$$
\mathrm{N}_{\|} \leq 7.5\left(\frac{1 \mathrm{keV}}{\mathrm{T}_{\mathrm{e}}}\right)^{1 / 2},
$$

which is a significant restriction if $\mathrm{T}_{\mathrm{e}} \geq 5 \mathrm{keV}$ at which temperature $\mathrm{N}_{\|}<3.3$. The dependence of $\mathrm{N}_{\|}$on nonlinear processes comes about because the group velocity is inversely proportional to the wavenumber. The energy of the slow wave is related to the input power by the relation

$$
\omega \frac{\partial \varepsilon}{\partial \omega} \frac{E^{2}}{8 \pi} v_{g x}=P
$$

so that a shorter wavelength wave will assume larger electric fields for the same input power. The threshold for nonlinear process depends, of course, on the magnitude of the electric field of the primary wave and thus any unwanted nonlinear absorption imposes a restriction on $\mathrm{N}_{\|}$for a given input power. 
It is instructive to evaluate the optimum $\mathrm{N}_{\|}$that produces the best coupling for both the fast and slow wave using Eqs. (70) and (71). The optimum $\mathrm{N}_{\|}$is then given by

$$
\begin{aligned}
\mathrm{N}_{11}^{2} & =1+\frac{\beta^{6}}{n_{p}^{2}} \\
& =1+168\left(\frac{50 \mathrm{~cm}}{a}\right)^{2}\left(\frac{m_{i}}{m_{p}}\right)^{2}\left(\frac{1 G I I}{f}\right)^{2} w^{4},
\end{aligned}
$$

for the slow wave and

$$
\begin{aligned}
\mathrm{N}_{\| 1}^{2} & =1+\frac{\beta^{6}}{\eta_{f}^{2}} \\
& =1+3.75\left(\frac{\mathrm{m}_{i}}{\mathrm{~m}_{\mathrm{p}}}\right)^{2}\left(\frac{50 \mathrm{~cm}}{\mathrm{a}}\right)^{2}\left(\frac{50 \mathrm{kG}}{\mathrm{B}}\right)^{2} \mathrm{w}^{4}
\end{aligned}
$$

for the fast wave. The optimum $\mathrm{N}_{\|}$for slow waves lies outside the range where the small argument expansion of Airey functions is valid; for fast waves, the optimum $\mathrm{N}_{\|}$may be obtainable for special cases. We note, however, that Eq. (76) gives a higher value of $N_{\|}$than is allowed by Eq. (72); i.e., the condition $\left|z_{f}\right| \ll 1$, for the parameters $m_{p}=m_{i}, a=50 \mathrm{~cm}$, and $B=50 \mathrm{kG}$. Inclusion of the full Airey function variation in the resistive impedance has a:strong effect on the results as is evident in 
Figs. 4 and 5 especially for the fast wave. Figure 5 shows that the restriction imposed by Eq. (72) is a strong one. Thus, fast wave launching requires strong magnetic fields in order to reduce $\mathrm{N}_{\|}^{*}$ below the limit imposed by (72). Moreover, the window in $\mathrm{N}_{\|}$is so narrow that a well-defined $\mathrm{N}_{\|}$is necessary to effectively couple to this wave.

The coupling of the source to the eigenmodes deserves some comment. For simplicity, we assume $j_{\|}=0$, and thus, the electric field at the current source is

$E_{y}=-\frac{4 \pi i \omega}{2 \omega^{2} N_{o}} j_{y}\left\{1+\exp \left[-2 N_{o}\left(\xi_{a}-\xi_{w}\right)\right]\left[\left(N_{y}^{2}-1\right) y_{22}+N_{\|} N_{y} Y_{21}\right]\right\}$

A pole of $y_{22}$ and $y_{21}$ in the limit $\left|z_{s}\right| \ll 1$ and $\left|z_{f}\right| \ll 1$, occurs for the eigenvalue condition

$\tan \left(\Phi+\alpha_{N}\right)=\frac{4}{\sqrt{3}} \frac{\beta^{2}+N_{0}^{2} \lambda_{s}^{-1} \lambda_{f}^{-1}+\beta\left(N_{o} / 2\right)\left(\lambda_{s}^{-1}+\lambda_{f}^{-1}\right)}{2 \beta N_{0}\left(\lambda_{s}^{-1}-\lambda_{f}^{-1}\right)} \equiv \tan \Phi_{0}$.

where $\alpha_{N}=\sin ^{-1}\left(N_{Y} / N_{0}\right)$. Equations (68) - (70) show that $\lambda_{s}>\lambda_{f} \sim 1$ and that the right hand side of Eq. (78) is of the order of one and varies little as a function of $\mathrm{N}_{\|}$. Equation (78) is satisfied by $\Phi_{n}=\Phi_{0}-\alpha_{N}+2 \pi n$. As Eqs. (7) and (43) 
make clear, $\Phi$ is a rapidly varying function of $N_{1}$ because $\omega \ll \omega_{\text {pe }}$ so the eigenvalues $\mathrm{N}_{\| \mathrm{n}}$ are densely packed; i.e., $\left|\mathrm{N}_{\| \mathrm{n}}-: \mathrm{N}_{\| \mathrm{n}+1}\right| / \mathrm{N}_{\| \mathrm{n}} \ll 1$. As a specific example, let us consider the resistive impedance of the finite current source,

$$
\begin{aligned}
j_{Y}(x, y, z) & =\hat{j} \exp \left(i k_{0} z-i \omega t\right),|z|<a \\
& =0 \quad,|z|>a
\end{aligned}
$$

due to the generation of eigenmodes. The fourier spectrum of this source

$$
j_{y}\left(x_{w}, \underline{k}\right)=\hat{j} \frac{\sin \left(k_{\|}-k_{o}\right) a}{\left(k_{11}-k_{0}\right) \pi}
$$

can be used in Eq. (77) to calculate the field in real space,

$$
\begin{aligned}
E_{y}\left(\xi_{w}, y, z\right)= & -\frac{4 \pi i \omega}{2 \omega^{2} \pi} \hat{j} \int_{-\infty}^{-\infty} \frac{d k_{\|}}{\sqrt{N_{\| !}^{2}-1}}\left\{1-\exp \left[-2 N_{0}\left(\xi_{a}-\xi_{w}\right)\right] y_{22}\right\} \\
& \exp \left(i k_{\|} t-i \omega t\right) \frac{\sin \left(k_{\|}-k_{0}\right) a}{\left(k_{\| 1}-k_{o}\right)},
\end{aligned}
$$

where we set $\mathrm{k}_{\mathrm{y}}=0$ in the integral. The resistive contributions come from the second term and are dominated by the eigenmode poles. The total field is the sum of these contributions. 
Neglecting the exponential factor and keeping only the second term, we obtain

$$
\begin{aligned}
E_{y}\left(\xi_{w}, y, z\right)= & -\frac{2 \pi i \omega}{\omega}\left(\sum_{m=-\infty}^{-1}, z_{m} \exp \left[-i\left(k_{0}-k_{m}\right)(z-a)\right]\right. \\
& \left.+\sum_{m=1}^{\infty} z_{m} \exp \left[-i\left(k_{0}-k_{m}\right)(z+a)\right]\right)
\end{aligned}
$$

where

$$
z_{m}=\left|\frac{d \Phi}{d k_{11}}\right|_{k_{l l}=k_{m}}^{-1}\left(\frac{\left(\Phi-\Phi_{m}\right) y_{22}}{\left(k_{m}-k_{o}\right)}\right)_{\Phi=\Phi_{m}}
$$

and $\mathrm{k}_{\mathrm{m}}$ are the values of $\mathrm{k}_{\|}$that satisfy the eigenvalue condition (78) and $\mathrm{k}_{-\mathrm{m}}=-\mathrm{k}_{\mathrm{m}}$. Using (79) and (81), we find the impedance of this source is proportional to $z_{m}$. We evaluate $z_{m}$

$$
z_{m}=\left|\frac{d k_{l l}}{d \Phi_{m}}\right| \frac{1}{\left(k_{m}-k_{o}\right)} \frac{\beta^{2}\left(1+v_{s}^{2}-v_{f}^{2}\right)+\left(N_{o}^{2} \nu_{f} \nu_{s}+N_{o} \beta\left(\nu_{s}+v_{f}\right) / 2\right)\left(\nu_{s}^{2}-v_{f}^{2}-1\right)}{2 \beta N_{o}\left(\nu_{s}-v_{f}\right) \sqrt{3}\left(1+\tan ^{2} \Phi_{o}\right)}
$$

where $\nu_{s}=\lambda_{s}^{-1}$ and $\nu_{f}=\lambda_{f}^{-1}$. Since $\left|d \Phi_{m} / d k_{\|}\right|\left|k_{m}-k_{o}\right|>>1$, 
and the other term is $\sim 1$, the impedance is in fact not large and coupling to these modes is not very effective.

We have already mentioned the limits that nonlinear parametric instabilities put on the parallel index of refraction to keep the electric fields of the wave below threshold. Another nonlinear effect, profile modification, comes into play near the slow wave cutoff. Large electric and magnetic fields, result from introducing between $(0.1 \div 1)$ MW of radiation to the plasma. Near the slow wave cutoff, the density and temperature are low enough that $\mathrm{E}^{2} / 8 \pi \mathrm{nT} \mathrm{T}_{\mathrm{e}}>1$. As a result, one would expect that the density profile will be steepened until the gradients are comparable to the electron Debye length [11]. How will this effect modify the results of our analysis? For the model presented here and $\mathrm{T}_{\mathrm{e}}=50 \mathrm{eV}$ at the slow wave cutoff, $\lambda_{\mathrm{De}} \simeq$ $2.2 \times 10^{-2} \mathrm{~cm}$ if $\mathrm{n}=10^{14} \mathrm{~cm}^{-3}$ at the center whereas, we use $\mathrm{L}_{\mathrm{p}} \simeq\left(\mathrm{m}_{\mathrm{e}} / \mathrm{m}_{\mathrm{i}}\right)(\mathrm{a} / 2)-1.4 \times 10^{-2} \mathrm{~cm}$. Thus, no great change will be produced by radiation pressure effects on the profile.

It should be clear from Table I that strong magnetic fields favor lower hybrid heating because the $\mathrm{N}_{\|}$required for accessibility is reduced. Penetration of the evanescent layer is no problem for the slow wave and the reflection coefficient for incident plane waves is quite insensitive to the density gradient scale length.for slow wave launching. The upper limit on the range of $\mathrm{N}_{\| l}$ for the slow wave is provided by avoidance of strong linear electron Landau damping $[c . f .,(76)]$ or nonlinear effects 
in the outer regions. If $\omega \geq 2 \omega_{1 h}$ and $\omega_{p e}>\omega_{c e}$, accessibility is difficult for the slow wave once the electron temperature of the outer regions exceeds $5 \mathrm{keV}$ for larger tokamaks (a $>\mathrm{lm}$ ). The penalty paid for a narrow band of wavenumbers is a large number of waveguides in the phased array as is evident in Eq. (65). The prospects improve considerably once $\omega_{\text {pe }}<\omega_{c e}$ and if $\omega \leqslant 2 \omega_{1 h}$, the range $2 \leqslant N_{l l} \lesssim 3$ is accessible. To judge the limitations imposed by nonlinear effects, we refer the reader to the literature $[6],[7]$.

Plasma turbulence may affect the coupling resonance that occurs for the waves with $1<\mathrm{N}_{\|}<\mathrm{N}_{\|}^{*}$. It has been predicted theoretically [6] and observed experimentally [12] that, when low frequency drift wave fluctuations reach a level such that $|\delta \mathrm{n} / \mathrm{n}| \sim 0.05$, the incoming lower hybrid slow wave radiation can be effectively scattered into waves such that $k_{y} \rho_{i} \sim 1$. One consequence of this scattering is that the cutoffs for the resultant slow and fast waves are moved deeper into the plasma because

$$
N_{Y} \sim \frac{{ }^{(1)} \mathrm{ci}}{\omega}\left(\frac{\mathrm{m}_{i} \mathrm{c}^{2}}{\mathrm{~T}_{i}}\right)^{1 / 2}=\frac{22.4}{\Omega}\left(\frac{1 \mathrm{keV}}{\mathrm{T}_{i}}\right)^{1 / 2},
$$

and thus using Eqs. (5) and (6), we find,

$$
\omega_{\mathrm{pe}}^{2}\left(\mathrm{x}_{\mathrm{s}}\right) \simeq 11.7 \frac{\omega \omega_{\mathrm{ce}}}{\Omega}\left(\frac{\mathrm{m}_{\mathrm{p}}}{\mathrm{m}_{1}}\right)^{1 / 2}\left(\frac{1 \mathrm{keV}}{\mathrm{T}_{1}}\right)
$$

and 
$\omega_{\mathrm{pe}}^{2}\left(\mathrm{x}_{\mathrm{f}}\right) \simeq 2 \dot{2} \cdot 36: \frac{{ }^{\prime} \omega_{\mathrm{ce}}}{\Omega}\left(\mathrm{N}_{\|}^{2}-1\right)^{-1 / 2}\left(\frac{1 \mathrm{keV}}{\mathrm{T}_{\mathrm{i}}}\right)^{1 / 2}$

At such large cutoff densities, the wave is effectively trapped in the plasma and the resistive impedance will decrease. As a result, an effective damping rate broadens the resonance and prevents the buildup of large electric fields in the low density rey Lun.

Plasma heating by the fast wave does present some difficulty. The fast wave does not have a strong linear damping. However, since the wave has a faster radial group velocity, the electric fields associated with the wave are smaller for the same input power. Thus, nonlinear effects; which occur at a threshold electric field, may be easier to avoid. The main difficulty in launching the wave is penetration of the evanescent region between the antenna and the fast wave cutoff. Penetration of this region imposes a severe restriction on $\mathrm{N}_{\|}[\mathrm{c} . \mathrm{f} .,(72)]$ so that only the range $1.2<\mathrm{N}_{\|}<1.5$ is accessible if $\omega=\omega_{1 \mathrm{~h}}$. The inclusion of the $k_{y}(\mathrm{dn} / \mathrm{dx})$ term in the equations near the fast wave cutoff will raise the upper limit somewhat if an antenna that launches a traveling wave such that $k_{y}\left(d_{n} / d x\right)<0$ is used. Overall, lower hybrid heating works best in small $(a<50 \mathrm{~cm}$ ), dense $\left(n>i 0^{14}\right.$ ) plasmas with strong magnetic fields ( $\left.\omega_{c e}>\omega_{p e}\right)$. 


\section{ACKNOWLEDGMENTS}

Beneficial discussions were held with Drs. S. Bernabei, M. Brambilla, W. Hooke, G. Morales, and F. Paoloni.

This work was supported by United States Energy Research and Development Administration Contract EY-76-C-02-3073. 


\section{REFERENCES}

1. W. M. Hooke, S. Bernabei, Phys. Rev. Lett. 29, 1218 (1972);

T. K. Chu, S. Bernabei, R. W. Motley, Phys. Rev. Lett. 31 , 211 (1973): R. P. H. Chang, M. Porkolab, Phys. Rev. Lett. $\underline{32}, 1117(1974)$.

2. V. V. Allikaev, Yu. I. Arsen'ev, G. A. Bobrovskịi, V. I. Poznyuk, K. A. nazumoua; aind Yu. A. Sukuluv, zh. Tiekh. Hiz. 45, 523 (1975) [Sov. Phýs. Tech. Phys. 20, 327 (1975)].

M. Porkolab, S. Bernabei, W. M. Hooke, R. W. Motley, and T. Nagashima, Phys. Rev. Lètt. 38,230 (1977).

W. Hess, P. Blanc, G. Ichtchenko, P. Javel, P. Lallia, C. Mahn, T. K. Nguyen, W. Ohlendorf, G. W. Pacher, H. D. Pacher, S. Takamura, G. Tonon, and J. G. Wegrowe, Bull. Am. Phys. Soc. $21,1107,5 \mathrm{D}-3(1976)$.

S. Takamura and P. Javel, "Observation of Turbulent spectra during the Lower Hybrid Heating in the Wega Tokamak." EUR-CEA-FC-863, November, 1976.

3. S. Bernabei, M. A. Heald, W. H. Hooke, F. Paoloni, Phys. Rev. Lett. 34,866 (1975). 
4. M. Brambilla, Nucl. Fusion 16, 47 (1976); M. Brambilla, Nucl. Fusion 14,327 (1974).

5. R. J. Briggs, R. R. Parker, Phys. Rev. Lett. 29, 852 (1972); P. M. Bellan and M. Porkolab, Phys. Fluids 17, 1592 (1974).

6. M. Porkolab, Phys. Fluids 17, 1432 (1975); R. L. Berger, L. Chen, P. K. Kaw, and F. W. Perkins, PPPL-1308 (to be published in Phys. Fluids 1977).

7. G. J. Morales and Y. C. Lee, Phys. Rev. Lett. $\underline{35}, 930$ (1975).

8. T. E. Stix, The Theory of Plasma Waves, McGraw Hill, New York, 1962; V. E. Golant; Sov. Phys. Tech. Phys. 16, 1980 (1972).

9. M. Brambilla, in Symposium on Plasma Heating in Toroidal Devices, Varenna, 1976.

Yu. F. Baranov and O. N. Scherbinin, Proc. 3rd International Meeting on Theoretical and Experimental Aspects of Heating of Toroidal Plasma, p. 131.

10. M. Abramowitz and I. Stegan, Handbook of Mathematical Functions, Dover Publications, Inc., New York, 1965, p. 446.

11. K. Whang and G. J. Morales, Bull. Am. Phys. Soc. 21, 1093, $4 F-3(1976)$.

12. P. M. Bellan and K. L. Wong, "The Effect of Density Fluctuations on Lower Hybrid Resonance Cone Propagation," Princeton Plasma Physics Lab Report \#1314, April, 1977. 
Table I

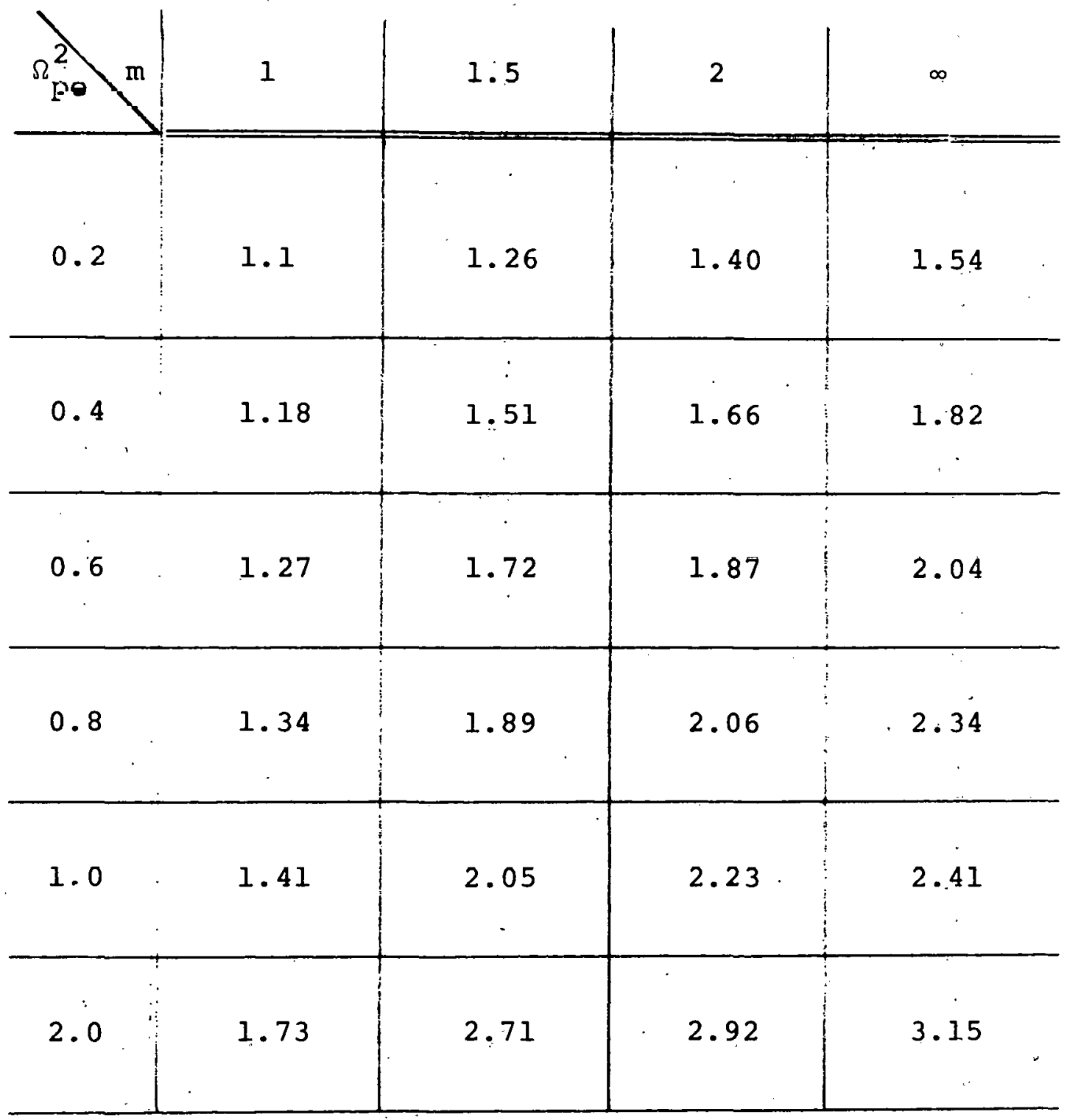




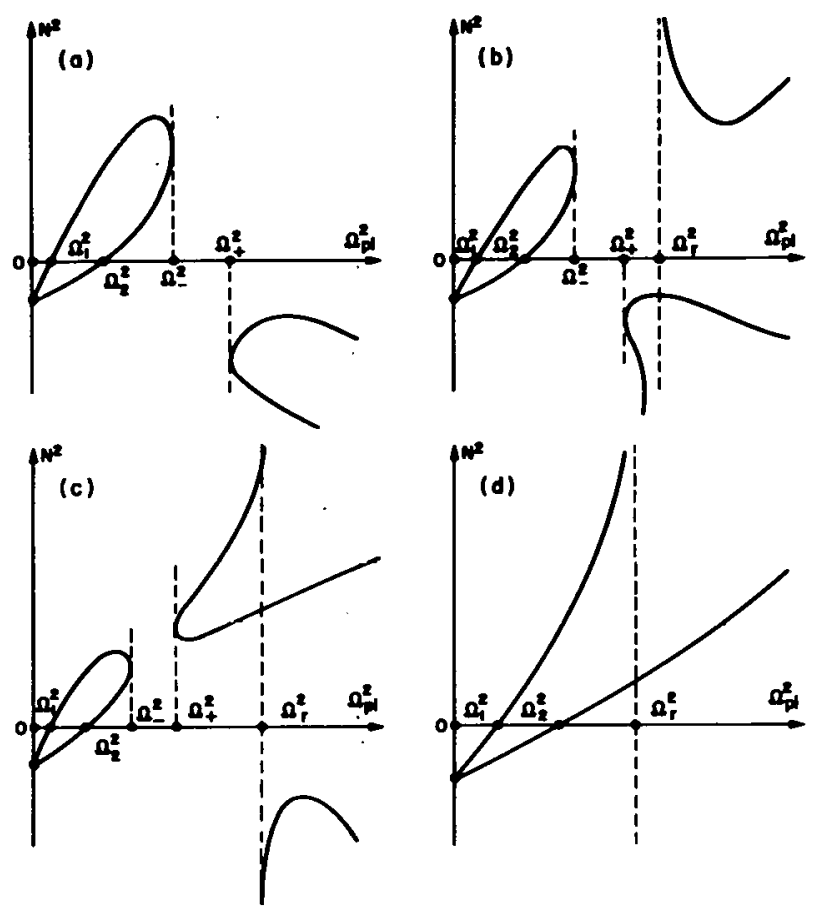

FIGURE I

772390

Fig. 1. Schematic representation of the two real roots of the cold plasma depression relation, $\Omega^{2} \equiv \omega^{2} / \omega_{c e} e_{c i}$
(a) $\Omega>1$;
(b) $2^{-1 / 2}<\Omega<1$ and $\mathrm{N}_{\|}^{2}<\Omega^{2} /\left(1-\Omega^{2}\right)$;
(c) $2^{-1 / 2}<\Omega<1$ and $\Omega^{2} /\left(1-\Omega^{2}\right)<N_{\|}^{2}<\left(1-\Omega^{2}\right)$;
(d) $\Omega<1, \mathrm{~N}_{\|}^{2}>\left(1-\Omega^{2}\right)^{-1}$;

$\mathrm{N}^{2}$ is complex between $\Omega_{-}^{2}$ and $\Omega_{+}^{2}$, where $\Omega_{ \pm}^{2}=\omega_{ \pm}^{2} / \omega_{\mathrm{Ce}} \omega_{\mathrm{ci}}$. The slow wave undergoes resonance at $\Omega_{\text {pe }}^{2}=\Omega_{r}^{2}=\Omega^{2} /\left(1-\Omega^{2}\right) \equiv$ $\omega_{r}^{2} / \omega_{c e} \omega_{c i}$. The slow and fast wave cutoffs occur for $\Omega_{p e}^{2}=\Omega_{1}^{2}$ and $\Omega_{\mathrm{pe}}^{2}=\Omega_{2}^{2}$ Iespeclively. 


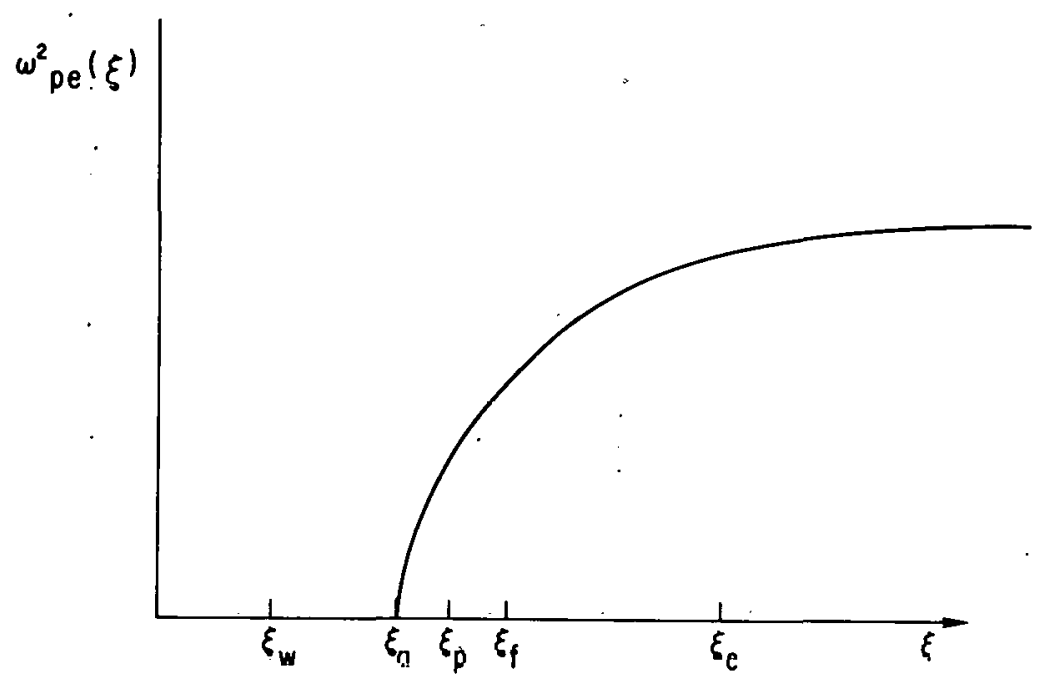

772377

Fig. 2. Model of density profile used to sompute wave reflection coefficients. The positions of the antenna, $\xi_{\text {, the }}$ plasma radius, $\xi$, the slow wave cutoff, $\xi$, the fast wave cutoff, $\xi_{f}$, and the mode conversion point, $\xi_{e} p^{\prime}$ are indicated. Here $\bar{\xi}=\omega \mathrm{X} / \mathrm{C}$.

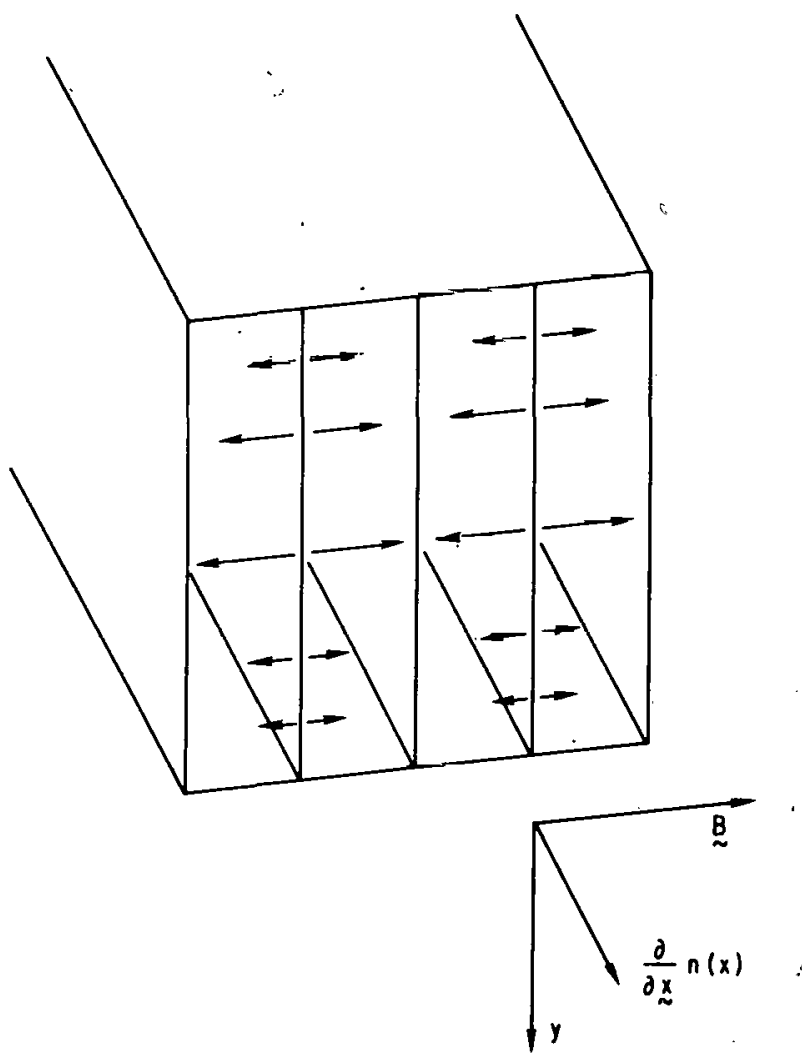

772391

Fig. 3. A schematic of a waveguide array excited in the $\mathrm{TE}_{10}$ mode such that the electric field is aligned with the magnetic field. Such an array launches a slow wave. The orientation of the waveguide with respecl to the plasma density gradient, the magnetic field, and the $y$ direction are shown in the inset. 


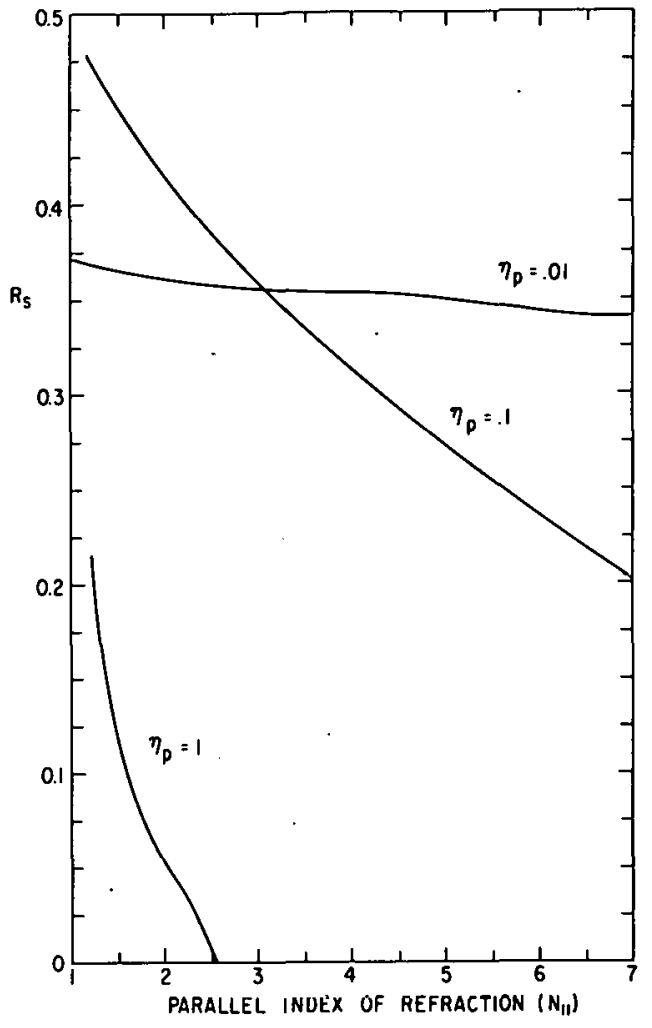

772380

Fig. 4. The resistive impedance for the slow wave $\mathrm{R}_{\mathrm{S}}$, including the full Airey function dependence, as a function of $N \|>N^{*}$ for $\mathrm{N}_{\mathrm{y}}=0$. The curves are labeled by the value of $n_{p}$ defined in Eq. (68).

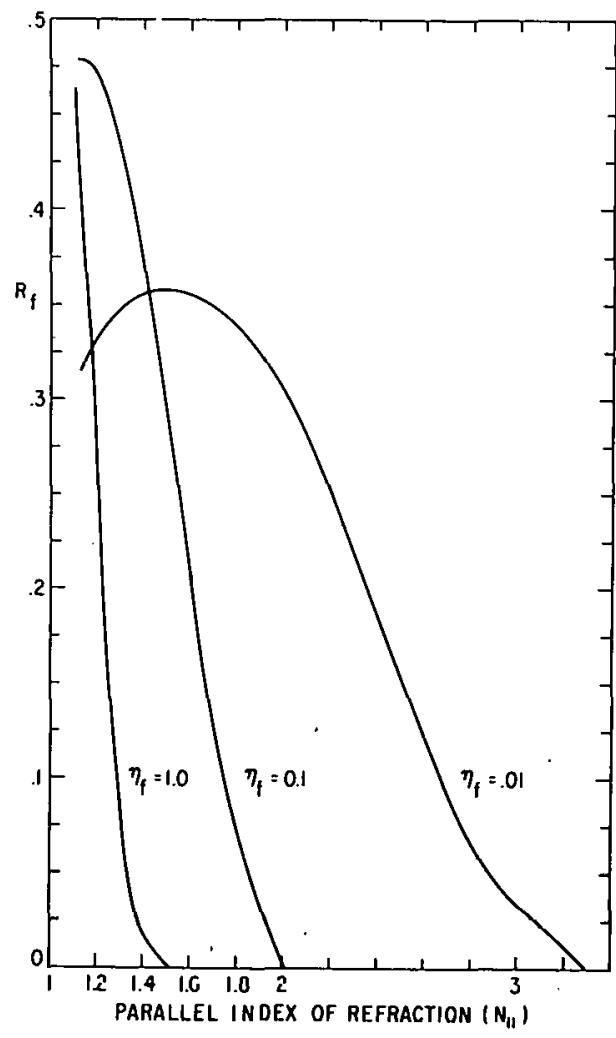

772381

Fig. 5. The resistive impedance for the fast wave $R_{f}$, including the full Airey function dependence, as a function of $\mathrm{N} \|>\mathrm{N}^{*}$ for $\mathrm{N}_{\mathrm{y}}=0$. The curves are labeled by the value of $n_{f}$ defined in Eq. (69). 